\title{
A General Inertial Projection-Type Algorithm for Solving Equilibrium Problem in Hilbert Spaces with Applications in Fixed-Point Problems
}

\author{
Nopparat Wairojjana ${ }^{1}{ }^{(\mathbb{C}, \text { Habib ur Rehman }}{ }^{2}{ }^{(}$, Manuel De la Sen ${ }^{3, *(1)}$ \\ and Nuttapol Pakkaranang ${ }^{2}$ D \\ 1 Applied Mathematics Program, Faculty of Science and Technology, \\ Valaya Alongkorn Rajabhat University under the Royal Patronage (VRU), 1 Moo 20 Phaholyothin Road, \\ Klong Neung, Klong Luang, Pathumthani 13180, Thailand; nopparat@vru.ac.th \\ 2 KMUTTFixed Point Research Laboratory, KMUTT-Fixed Point Theory and Applications Research Group, \\ SCL 802 Fixed Point Laboratory, Department of Mathematics, Faculty of Science, \\ King Mongkut's University of Technology Thonburi (KMUTT), 126 Pracha-Uthit Road, Bang Mod, \\ Thrung Khru, Bangkok 10140, Thailand; habib.rehman@mail.kmutt.ac.th (H.u.R.); \\ nuttapol.pak@mail.kmutt.ac.th (N.P.) \\ 3 Institute of Research and Development of Processes IIDP, University of the Basque Country, \\ Leioa 48940, Spain \\ * Correspondence: manuel.delasen@ehu.eus; Tel.:+34-94-601-2548
}

Received: 30 July 2020; Accepted: 28 August 2020; Published: 31 August 2020

\begin{abstract}
A plethora of applications from mathematical programming, such as minimax, and mathematical programming, penalization, fixed point to mention a few can be framed as equilibrium problems. Most of the techniques for solving such problems involve iterative methods that is why, in this paper, we introduced a new extragradient-like method to solve equilibrium problems in real Hilbert spaces with a Lipschitz-type condition on a bifunction. The advantage of a method is a variable stepsize formula that is updated on each iteration based on the previous iterations. The method also operates without the previous information of the Lipschitz-type constants. The weak convergence of the method is established by taking mild conditions on a bifunction. For application, fixed-point theorems that involve strict pseudocontraction and results for pseudomonotone variational inequalities are studied. We have reported various numerical results to show the numerical behaviour of the proposed method and correlate it with existing ones.
\end{abstract}

Keywords: convex optimization; pseudomonotone bifunction; equilibrium problems; variational inequality problems; weak convergence; fixed point problems

\section{Introduction}

For a nonempty, closed and convex subset $\mathcal{K}$ of a real Hilbert space $\mathcal{E}$ and $f: \mathcal{E} \times \mathcal{E} \rightarrow \mathcal{R}$ is a bifunction with $f\left(p_{1}, p_{1}\right)=0$, for each $p_{1} \in \mathcal{K}$. A equilibrium problem [1,2] for $f$ on the set $\mathcal{K}$ is defined in the following way:

$$
\text { Find } \wp^{*} \in \mathcal{K} \text { such that } f\left(\wp^{*}, p_{1}\right) \geq 0, \forall p_{1} \in \mathcal{K} \text {. }
$$

The problem (1) is very general, it includes many problems, such as fixed point problems, variational inequalities problems, the optimization problems, the Nash equilibrium of non-cooperative games, the complementarity problems, the saddle point problems, and the vector optimization problem (for further details see $[1,3,4])$. The equilibrium problem is also considered as the famous Ky Fan inequality [2]. This above-defined particular format of an equilibrium problem (1) is initiated by 
Muu and Oettli [5] in 1992 and further investigation on its theoretical properties studied by Blum and Oettli [1]. The construction of new optimization-based methods and the modification and extension of existing methods, as well as the examination of their convergence analysis, is an important research direction in equilibrium problem theory. Many methods have been developed over the last few years to numerically solve the equilibrium problems in both finite and infinite dimensional Hilbert spaces, i.e., the extragradient algorithms [6-14] subgradient algorithms [15-21] inertial methods [22-25], and others in [26-34].

In particular, a proximal method [35] is an efficient way to solve equilibrium problems that are equivalent to solving minimization problems on each step. This approach is also considered as the two-step extragradient-like method in [6], because of the early contribution of the Korpelevich [36] extragradient method to solve the saddle point problems. More precisely, Tran et al. introduced a method in [6], in which an iterative sequence $\left\{u_{n+1}\right\}$ was generated in the following manner:

$$
\left\{\begin{array}{l}
u_{n} \in \mathcal{K}, \\
v_{n}=\arg \min \left\{\xi f\left(u_{n}, y\right)+\frac{1}{2}\left\|u_{n}-y\right\|^{2}: y \in \mathcal{K}\right\} \\
u_{n+1}=\arg \min \left\{\xi f\left(v_{n}, y\right)+\frac{1}{2}\left\|u_{n}-y\right\|^{2}: y \in \mathcal{K}\right\}
\end{array}\right.
$$

where $0<\xi<\min \left\{\frac{1}{2 k_{1}}, \frac{1}{2 k_{2}}\right\}$ and $k_{1}, k_{2}$ are Lipschitz constants. Moreover, $\underset{y \in \mathcal{K}}{\arg \min } f(x)$ is the value of $x$ in set $\mathcal{K}$ for which $f(x)$ attains it's minimum. The iterative sequence generated from the above-described method provides a weak convergent iterative sequence and in order to operate it, previous knowledge of the Lipschitz-like constants are required. These Lipschitz-type constants are normally unknown or hard to evaluate. In order to overcome this situation, Hieu et al. [12] introduced an extension of the method in [37] to solve the problems of equilibrium in the following manner: let $[t]_{+}:=\max \{t, 0\}$ and choose $u_{0} \in \mathcal{K}, \mu \in(0,1)$ with $\xi_{0}>0$, such that

$$
\left\{\begin{array}{l}
v_{n}=\arg \min \left\{\xi_{n} f\left(u_{n}, y\right)+\frac{1}{2}\left\|u_{n}-y\right\|^{2}: y \in \mathcal{K}\right\}, \\
u_{n+1}=\arg \min \left\{\xi_{n} f\left(v_{n}, y\right)+\frac{1}{2}\left\|u_{n}-y\right\|^{2}: y \in \mathcal{K}\right\},
\end{array}\right.
$$

where the stepsize sequence $\left\{\xi_{n}\right\}$ is updated in the following way:

$$
\xi_{n+1}=\min \left\{\xi_{n}, \frac{\mu\left(\left\|u_{n}-v_{n}\right\|^{2}+\left\|u_{n+1}-v_{n}\right\|^{2}\right)}{2\left[f\left(u_{n}, u_{n+1}\right)-f\left(u_{n}, v_{n}\right)-f\left(v_{n}, u_{n+1}\right)\right]_{+}}\right\}
$$

Recently, Vinh and Muu proposed an inertial iterative algorithm in [38] to solve a pseudomonotone equilibrium problem. The key contribution is an inertial factor in the method that used to enhance the convergence speed of the iterative sequence. The iterative sequence $\left\{u_{n}\right\}$ was defined in the following manner:

(i) Choose $u_{-1}, u_{0} \in \mathcal{K}, \theta \in[0,1), 0<\xi<\min \left\{\frac{1}{2 k_{1}}, \frac{1}{2 k_{2}}\right\}$ where a sequence $\left\{\rho_{n}\right\} \subset[0,+\infty)$ is satisfies the following conditions:

$$
\sum_{n=0}^{+\infty} \rho_{n}<+\infty
$$

(ii) Choose $\theta_{n}$ satisfying $0 \leq \theta_{n} \leq \overline{\theta_{n}}$ and

$$
\overline{\theta_{n}}= \begin{cases}\min \left\{\theta, \frac{\rho_{n}}{\left\|u_{n}-u_{n-1}\right\|}\right\} & \text { if } u_{n} \neq u_{n-1} \\ \theta & \text { else. }\end{cases}
$$


(iii) Compute

$$
\left\{\begin{array}{l}
\varrho_{n}=u_{n}+\theta_{n}\left(u_{n}-u_{n-1}\right) \\
v_{n}=\arg \min \left\{\xi f\left(\varrho_{n}, y\right)+\frac{1}{2}\left\|\varrho_{n}-y\right\|^{2}: y \in \mathcal{K}\right\} \\
u_{n+1}=\arg \min \left\{\xi f\left(v_{n}, y\right)+\frac{1}{2}\left\|\varrho_{n}-y\right\|^{2}: y \in \mathcal{K}\right\} .
\end{array}\right.
$$

Recently, another efficient inertial algorithm proposed by Hieu et al. in [39] as follows: let $u_{n-1}, u_{n}, v_{n} \in \mathcal{K}, \theta \in[0,1), 0<\xi \leq \frac{1}{2 k_{2}+8 k_{1}}$ and the sequence $\left\{u_{n}\right\}$ was defined in the following manner:

$$
\left\{\begin{array}{l}
\varrho_{n}=u_{n}+\theta\left(u_{n}-u_{n-1}\right), \\
u_{n+1}=\arg \min \left\{\xi f\left(v_{n}, y\right)+\frac{1}{2}\left\|\varrho_{n}-y\right\|^{2}: y \in \mathcal{K}\right\} \\
\varrho_{n+1}=u_{n+1}+\theta\left(u_{n+1}-u_{n}\right) \\
v_{n+1}=\arg \min \left\{\tilde{\zeta} f\left(v_{n}, y\right)+\frac{1}{2}\left\|\varrho_{n+1}-y\right\|^{2}: y \in \mathcal{K}\right\}
\end{array}\right.
$$

In this article, we concentrates on projection methods that are normally well-established and easy to execute due to their efficient numerical computation. Motivated by the works of [12,38], we formulate an inertial explicit subgradient extragradient method to solve the pseudomonotone equilibrium problem. These results can be seen as the modification of the methods appeared in $[6,12,38,39]$. Under certain mild conditions, a weak convergence theorem is proved regarding the iterative sequence of the algorithm. Moreover, experimental studies have documented that the designed method tends to be more efficient when compared to the existing methods that are presented in $[38,39]$.

The remainder of the paper has been arranged, as follows: Section 2 contains the elementary results used in this paper. Section 3 contains our main algorithm and proves their convergence. Sections 4 and 5 incorporate the applications of our main results. Section 6 carries out the numerical results that prove the computational effectiveness of our suggested method.

\section{Preliminaries}

Assume that $h: \mathcal{K} \rightarrow \mathcal{R}$ be a convex function on a nonempty, closed and convex subset $\mathcal{K}$ of a real Hilbert space $\mathcal{E}$ and subdifferential of a function $h$ at $p_{1} \in \mathcal{K}$ is defined by

$$
\partial h\left(p_{1}\right)=\left\{p_{3} \in \mathcal{E}: h\left(p_{2}\right)-h\left(p_{1}\right) \geq\left\langle p_{3}, p_{2}-p_{1}\right\rangle, \forall p_{2} \in \mathcal{K}\right\} .
$$

Assume that $\mathcal{K}$ be a nonempty, closed and convex subset of a real Hilbert space $\mathcal{E}$ and Normal cone of $\mathcal{K}$ at $p_{1} \in \mathcal{K}$ is defined by

$$
N_{\mathcal{K}}\left(p_{1}\right)=\left\{p_{3} \in \mathcal{E}:\left\langle p_{3}, p_{2}-p_{1}\right\rangle \leq 0, \forall p_{2} \in \mathcal{K}\right\} .
$$

A metric projection $P_{\mathcal{K}}\left(p_{1}\right)$ for $p_{1} \in \mathcal{E}$ onto a closed and convex subset $\mathcal{K}$ of $\mathcal{E}$ is defined by

$$
P_{\mathcal{K}}\left(p_{1}\right)=\arg \min \left\{\left\|p_{2}-p_{1}\right\|: p_{2} \in \mathcal{K}\right\} .
$$

Now, consider the following definitions of monotonicity a bifunction (see for details $[1,40]$ ). Assume that $f: \mathcal{E} \times \mathcal{E} \rightarrow \mathcal{R}$ on $\mathcal{K}$ for $\gamma>0$ is said to be

$\gamma$-strongly monotone if

$$
f\left(p_{1}, p_{2}\right)+f\left(p_{2}, p_{1}\right) \leq-\gamma\left\|p_{1}-p_{2}\right\|^{2}, \forall p_{1}, p_{2} \in \mathcal{K} ;
$$

(2) monotone if

$$
f\left(p_{1}, p_{2}\right)+f\left(p_{2}, p_{1}\right) \leq 0, \forall p_{1}, p_{2} \in \mathcal{K}
$$


(3) $\gamma$-strongly pseudomonotone if

$$
f\left(p_{1}, p_{2}\right) \geq 0 \Longrightarrow f\left(p_{2}, p_{1}\right) \leq-\gamma\left\|p_{1}-p_{2}\right\|^{2}, \forall p_{1}, p_{2} \in \mathcal{K}
$$

(4) pseudomonotone if

$$
f\left(p_{1}, p_{2}\right) \geq 0 \Longrightarrow f\left(p_{2}, p_{1}\right) \leq 0, \forall p_{1}, p_{2} \in \mathcal{K} .
$$

We have the following implications from the above definitions:

$$
(1) \Longrightarrow(2) \Longrightarrow(4) \text { and }(1) \Longrightarrow(3) \Longrightarrow(4) \text {. }
$$

In general, the converses are not true. Suppose that $f: \mathcal{E} \times \mathcal{E} \rightarrow \mathcal{R}$ satisfy the Lipschitz-type condition [41] on a set $\mathcal{K}$ if there exist two constants $k_{1}, k_{2}>0$, such that

$$
f\left(p_{1}, p_{2}\right)+f\left(p_{2}, p_{3}\right)+k_{1}\left\|p_{1}-p_{2}\right\|^{2}+k_{2}\left\|p_{2}-p_{3}\right\|^{2} \geq f\left(p_{1}, p_{3}\right), \forall p_{1}, p_{2}, p_{3} \in \mathcal{K}
$$

Lemma 1 ([42]). Suppose $\mathcal{K}$ be a nonempty, closed and convex subset of $\mathcal{E}$ and $P_{\mathcal{K}}: \mathcal{E} \rightarrow \mathcal{K}$ is metric projection from $\mathcal{E}$ onto $\mathcal{K}$.

(i) Let $p_{1} \in \mathcal{K}$ and $p_{2} \in \mathcal{E}$, we have

$$
\left\|p_{1}-P_{\mathcal{K}}\left(p_{2}\right)\right\|^{2}+\left\|P_{\mathcal{K}}\left(p_{2}\right)-p_{2}\right\|^{2} \leq\left\|p_{1}-p_{2}\right\|^{2}
$$

(ii) $\quad p_{3}=P_{\mathcal{K}}\left(p_{1}\right)$ if and only if

$$
\left\langle p_{1}-p_{3}, p_{2}-p_{3}\right\rangle \leq 0, \forall p_{2} \in \mathcal{K}
$$

(iii) For any $p_{2} \in \mathcal{K}$ and $p_{1} \in \mathcal{E}$

$$
\left\|p_{1}-P_{\mathcal{K}}\left(p_{1}\right)\right\| \leq\left\|p_{1}-p_{2}\right\|
$$

Lemma $2([43,44])$. Assume that $h: \mathcal{K} \rightarrow \mathcal{R}$ be a convex, lower semicontinuous and subdifferentiable function on $\mathcal{K}$, where $\mathcal{K}$ is a nonempty, convex and closed subset of a Hilbert space $\mathcal{E}$. Subsequently, $p_{1} \in \mathcal{K}$ is minimizer of a function $h$ if and only if $0 \in \partial h\left(p_{1}\right)+N_{\mathcal{K}}\left(p_{1}\right)$, where $\partial h\left(p_{1}\right)$ and $N_{\mathcal{K}}\left(p_{1}\right)$ denotes the subdifferential of $h$ at $p_{1} \in \mathcal{K}$ and the normal cone of $\mathcal{K}$ at $p_{1}$, respectively.

Lemma 3 ([45]). Let $\left\{u_{n}\right\}$ be a sequence in $\mathcal{E}$ and $\mathcal{K} \subset \mathcal{E}$, such that the following conditions are satisfied:

(i) for every $u \in \mathcal{K}$, the $\lim _{n \rightarrow \infty}\left\|u_{n}-u\right\|$ exists;

(ii) each sequentially weak cluster limit point of the sequence $\left\{u_{n}\right\}$ belongs to $\mathcal{K}$.

Then, $\left\{u_{n}\right\}$ weakly converge to some element in $\mathcal{K}$.

Lemma 4 ([46]). Let $\left\{q_{n}\right\}$ and $\left\{p_{n}\right\}$ be sequences of non-negative real numbers satisfying $q_{n+1} \leq q_{n}+p_{n}$, for each $n \in \mathcal{N}$. If $\sum p_{n}<\infty$, then $\lim _{n \rightarrow \infty} q_{n}$ exists.

Lemma 5 ([47]). For every $p_{1}, p_{2} \in \mathcal{E}$ and $\zeta \in \mathcal{R}$, then

$$
\left\|\zeta p_{1}+(1-\zeta) p_{2}\right\|^{2}=\zeta\left\|p_{1}\right\|^{2}+(1-\zeta)\left\|p_{2}\right\|^{2}-\zeta(1-\zeta)\left\|p_{1}-p_{2}\right\|^{2}
$$

Suppose that bifunction $f$ satisfies the following conditions:

(f1) $f$ is pseudomonotone on $\mathcal{K}$ and $f\left(p_{2}, p_{2}\right)=0$, for every $p_{2} \in \mathcal{K}$;

(f2) $f$ satisfies the Lipschitz-type condition on $\mathcal{E}$ with constants $k_{1}>0$ and $k_{2}>0$;

(f3) $\quad \limsup _{n \rightarrow \infty} f\left(p_{n}, v\right) \leq f\left(p^{*}, v\right)$ for every $v \in \mathcal{K}$ and $\left\{p_{n}\right\} \subset \mathcal{K}$ satisfying $p_{n} \rightarrow p^{*}$;

(f4) $\quad f\left(p_{1},.\right)$ needs to be convex and subdifferentiable on $\mathcal{E}$ for all $p_{1} \in \mathcal{E}$. 


\section{The Modified Extragradient Algorithm for the Problem (1) and Its Convergence Analysis}

We provide a method consisting of two strongly convex minimization problems with an inertial term and an explicit stepsize formula that are being used to enhance the convergence rate of the iterative sequence and to make the algorithm independent of the Lipschitz constants. For the sake of simplicity in the presentation, we will use the notation $[t]_{+}=\max \{0, t\}$ and follow the conventions $\frac{0}{0}=+\infty$ and $\frac{a}{0}=+\infty(a \neq 0)$. The detailed method is provided below (Algorithm 1):

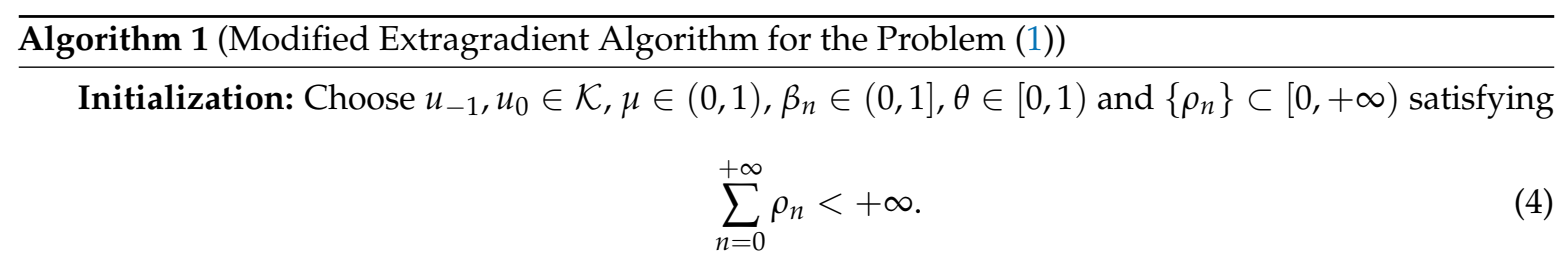

Iterative steps: Choose $\theta_{n}$ satisfying $0 \leq \theta_{n} \leq \overline{\theta_{n}}$ and

$$
\overline{\theta_{n}}= \begin{cases}\min \left\{\theta, \frac{\rho_{n}}{\left\|u_{n}-u_{n-1}\right\|}\right\} & \text { if } u_{n} \neq u_{n-1}, \\ \theta & \text { else. }\end{cases}
$$

Step 1: Compute

$$
v_{n}=\underset{y \in \mathcal{K}}{\arg \min }\left\{\xi_{n} f\left(\varrho_{n}, y\right)+\frac{1}{2}\left\|\varrho_{n}-y\right\|^{2}\right\},
$$

where $\varrho_{n}=u_{n}+\theta_{n}\left(u_{n}-u_{n-1}\right)$. If $\varrho_{n}=v_{n} ;$ STOP. Else, go to next step.

Step 2: Compute $u_{n+1}=\left(1-\beta_{n}\right) \varrho_{n}+\beta_{n} z_{n}$, where

$$
z_{n}=\underset{y \in \mathcal{K}}{\arg \min }\left\{\xi_{n} f\left(v_{n}, y\right)+\frac{1}{2}\left\|\varrho_{n}-y\right\|^{2}\right\} .
$$

Step 3: Update the stepsize in the following manner:

$$
\xi_{n+1}=\min \left\{\xi_{n}, \frac{\mu\left\|\varrho_{n}-v_{n}\right\|^{2}+\mu\left\|z_{n}-v_{n}\right\|^{2}}{2\left[f\left(\varrho_{n}, z_{n}\right)-f\left(\varrho_{n}, v_{n}\right)-f\left(v_{n}, z_{n}\right)\right]_{+}}\right\} .
$$

Put $n:=n+1$ and return to Iterative steps.

Lemma 6. The sequence $\left\{\xi_{n}\right\}$ is monotonically decreasing with a lower bound $\min \left\{\frac{\mu}{2 \max \left\{k_{1}, k_{2}\right\}}, \xi_{0}\right\}$ and it converges to $\xi>0$.

Proof. From the definition of sequence $\left\{\xi_{n}\right\}$ implies that sequence $\left\{\xi_{n}\right\}$ decreasing monotonically. It is given that $f$ satisfy the Lipschitz-type condition with $k_{1}$ and $k_{2}$. Let $f\left(\varrho_{n}, z_{n}\right)-f\left(\varrho_{n}, v_{n}\right)-f\left(v_{n}, z_{n}\right)>0$, such that

$$
\begin{aligned}
\frac{\mu\left(\left\|\varrho_{n}-v_{n}\right\|^{2}+\left\|z_{n}-v_{n}\right\|^{2}\right)}{2\left[f\left(\varrho_{n}, z_{n}\right)-f\left(\varrho_{n}, v_{n}\right)-f\left(v_{n}, z_{n}\right)\right]} & \geq \frac{\mu\left(\left\|\varrho_{n}-v_{n}\right\|^{2}+\left\|z_{n}-v_{n}\right\|^{2}\right)}{2\left[k_{1}\left\|\varrho_{n}-v_{n}\right\|^{2}+k_{2}\left\|z_{n}-v_{n}\right\|^{2}\right]} \\
& \geq \frac{\mu}{2 \max \left\{k_{1}, k_{2}\right\}} .
\end{aligned}
$$

The above implies that $\left\{\xi_{n}\right\}$ has a lower bound $\min \left\{\frac{\mu}{2 \max \left\{k_{1}, k_{2}\right\}}, \xi_{0}\right\}$. Moreover, there exists a fixed real number $\xi>0$, such that $\lim _{n \rightarrow \infty} \xi_{n}=\xi$. 
Remark 1. Because of the summability of $\sum_{n=0}^{+\infty} \rho_{n}$ and the expression (5) implies that

$$
\sum_{n=1}^{\infty} \theta_{n}\left\|u_{n}-u_{n-1}\right\| \leq \sum_{n=1}^{\infty} \overline{\theta_{n}}\left\|u_{n}-u_{n-1}\right\| \leq \sum_{n=1}^{\infty} \theta\left\|u_{n}-u_{n-1}\right\|<\infty
$$

that implies

$$
\lim _{n \rightarrow \infty} \theta\left\|u_{n}-u_{n-1}\right\|=0
$$

Lemma 7. Suppose that $f: \mathcal{E} \times \mathcal{E} \rightarrow \mathcal{R}$ be a bifunction satisfies the conditions (f1)-(f4). For each $\wp^{*} \in E P(f, \mathcal{K}) \neq \varnothing$, we have

$$
\left\|z_{n}-\wp^{*}\right\|^{2} \leq\left\|\varrho_{n}-\wp^{*}\right\|^{2}-\left(1-\frac{\mu \xi_{n}}{\xi_{n+1}}\right)\left\|\varrho_{n}-v_{n}\right\|^{2}-\left(1-\frac{\mu \xi_{n}}{\xi_{n+1}}\right)\left\|z_{n}-v_{n}\right\|^{2} .
$$

Proof. From the value of $z_{n}$, we have

$$
0 \in \partial_{2}\left\{\xi_{n} f\left(v_{n}, y\right)+\frac{1}{2}\left\|\varrho_{n}-y\right\|^{2}\right\}\left(z_{n}\right)+N_{\mathcal{K}}\left(z_{n}\right) .
$$

For some $\omega \in \partial f\left(v_{n}, z_{n}\right)$, there exists $\bar{\omega} \in N_{\mathcal{K}}\left(z_{n}\right)$, such that

$$
\xi_{n} \omega+z_{n}-\varrho_{n}+\bar{\omega}=0 .
$$

The above expression implies that

$$
\left\langle\varrho_{n}-z_{n}, y-z_{n}\right\rangle=\xi_{n}\left\langle\omega, y-z_{n}\right\rangle+\left\langle\bar{\omega}, y-z_{n}\right\rangle, \forall y \in \mathcal{K}
$$

For given $\bar{\omega} \in N_{\mathcal{K}}\left(z_{n}\right)$, imply that $\left\langle\bar{\omega}, y-z_{n}\right\rangle \leq 0, \forall y \in \mathcal{K}$. It provides that

$$
\left\langle\varrho_{n}-z_{n}, y-z_{n}\right\rangle \leq \xi_{n}\left\langle\omega, y-z_{n}\right\rangle, \forall y \in \mathcal{K}
$$

From $\omega \in \partial f\left(v_{n}, z_{n}\right)$, we have

$$
f\left(v_{n}, y\right)-f\left(v_{n}, z_{n}\right) \geq\left\langle\omega, y-z_{n}\right\rangle, \forall y \in \mathcal{E}
$$

Combining expressions (9) and (10) we obtain

$$
\xi_{n} f\left(v_{n}, y\right)-\xi_{n} f\left(v_{n}, z_{n}\right) \geq\left\langle\varrho_{n}-z_{n}, y-z_{n}\right\rangle, \forall y \in \mathcal{K}
$$

By substituting $y=\wp^{*}$ in (11), gives that

$$
\xi_{n} f\left(v_{n}, \wp^{*}\right)-\xi_{n} f\left(v_{n}, z_{n}\right) \geq\left\langle\varrho_{n}-z_{n}, \wp^{*}-z_{n}\right\rangle .
$$

Because $f\left(\wp^{*}, v_{n}\right) \geq 0$, then $f\left(v_{n}, \wp^{*}\right) \leq 0$, provides that

$$
\left\langle\varrho_{n}-z_{n}, z_{n}-\wp^{*}\right\rangle \geq \xi_{n} f\left(v_{n}, z_{n}\right) .
$$

From the formula of $\xi_{n+1}$, we obtain

$$
f\left(\varrho_{n}, z_{n}\right)-f\left(\varrho_{n}, v_{n}\right)-f\left(v_{n}, z_{n}\right) \leq \frac{\mu\left\|\varrho_{n}-v_{n}\right\|^{2}+\mu\left\|z_{n}-v_{n}\right\|^{2}}{2 \xi_{n+1}}
$$


From the expressions (13) and (14), we have

$$
\begin{aligned}
\left\langle\varrho_{n}-z_{n}, z_{n}-\wp^{*}\right\rangle \geq & \xi_{n}\left\{f\left(\varrho_{n}, z_{n}\right)-f\left(\varrho_{n}, v_{n}\right)\right\} \\
& -\frac{\mu \xi_{n}}{2 \xi_{n+1}}\left\|\varrho_{n}-v_{n}\right\|^{2}-\frac{\mu \xi_{n}}{2 \xi_{n+1}}\left\|z_{n}-v_{n}\right\|^{2} .
\end{aligned}
$$

Similar to expression (11), the value of $v_{n}$ gives that

$$
\xi_{n} f\left(\varrho_{n}, y\right)-\xi_{n} f\left(\varrho_{n}, v_{n}\right) \geq\left\langle\varrho_{n}-v_{n}, y-v_{n}\right\rangle, \forall y \in \mathcal{K} .
$$

By substituting $y=z_{n}$ in the above expression, we have

$$
\xi_{n}\left\{f\left(\varrho_{n}, z_{n}\right)-f\left(\varrho_{n}, v_{n}\right)\right\} \geq\left\langle\varrho_{n}-v_{n}, z_{n}-v_{n}\right\rangle .
$$

Combining the expressions (15) and (17), we obtain

$$
\begin{aligned}
\left\langle\varrho_{n}-z_{n}, z_{n}-\wp^{*}\right\rangle \geq & \left\langle\varrho_{n}-v_{n}, z_{n}-v_{n}\right\rangle \\
& -\frac{\mu \xi_{n}}{2 \xi_{n+1}}\left\|\varrho_{n}-v_{n}\right\|^{2}-\frac{\mu \xi_{n}}{2 \xi_{n+1}}\left\|z_{n}-v_{n}\right\|^{2} .
\end{aligned}
$$

We have the given formulas:

$$
\begin{gathered}
-2\left\langle\varrho_{n}-z_{n}, z_{n}-\wp^{*}\right\rangle=-\left\|\varrho_{n}-\wp^{*}\right\|^{2}+\left\|z_{n}-\varrho_{n}\right\|^{2}+\left\|z_{n}-\wp^{*}\right\|^{2} . \\
2\left\langle v_{n}-\varrho_{n}, v_{n}-z_{n}\right\rangle=\left\|\varrho_{n}-v_{n}\right\|^{2}+\left\|z_{n}-v_{n}\right\|^{2}-\left\|\varrho_{n}-z_{n}\right\|^{2} .
\end{gathered}
$$

The above expressions with (18), we have

$$
\left\|z_{n}-\wp^{*}\right\|^{2} \leq\left\|\varrho_{n}-\wp^{*}\right\|^{2}-\left(1-\frac{\mu \xi_{n}}{\xi_{n+1}}\right)\left\|\varrho_{n}-v_{n}\right\|^{2}-\left(1-\frac{\mu \xi_{n}}{\xi_{n+1}}\right)\left\|z_{n}-v_{n}\right\|^{2} .
$$

Theorem 1. Assume that $f: \mathcal{E} \times \mathcal{E} \rightarrow \mathcal{R}$ be a bifunction satisfies the conditions (f1)-(f4) and $\wp^{*}$ belongs to solution set $\operatorname{EP}(f, \mathcal{K})$. Subsequently, the sequences $\left\{\varrho_{n}\right\},\left\{v_{n}\right\},\left\{z_{n}\right\}$ and $\left\{u_{n}\right\}$ generated through Algorithm 1 weakly converges to $\wp^{*}$. In addition, $\lim _{n \rightarrow \infty} P_{E P(f, \mathcal{K})}\left(u_{n}\right)=\wp^{*}$.

Proof. By value of $u_{n+1}$ through Lemma 5, we obtain

$$
\begin{aligned}
\left\|u_{n+1}-\wp^{*}\right\|^{2} & =\left\|\left(1-\beta_{n}\right) \varrho_{n}+\beta_{n} z_{n}-\wp^{*}\right\|^{2} \\
& =\left\|\left(1-\beta_{n}\right)\left(\varrho_{n}-\wp^{*}\right)+\beta_{n}\left(z_{n}-\wp^{*}\right)\right\|^{2} \\
& =\left(1-\beta_{n}\right)\left\|\varrho_{n}-\wp^{*}\right\|^{2}+\beta_{n}\left\|z_{n}-\wp^{*}\right\|^{2}-\beta_{n}\left(1-\beta_{n}\right)\left\|\varrho_{n}-z_{n}\right\|^{2} \\
& \leq\left(1-\beta_{n}\right)\left\|\varrho_{n}-\wp^{*}\right\|^{2}+\beta_{n}\left\|z_{n}-\wp^{*}\right\|^{2} .
\end{aligned}
$$

By Lemma 7 and expression (19), we obtain

$$
\begin{aligned}
\left\|u_{n+1}-\wp^{*}\right\|^{2} \leq & \left\|\varrho_{n}-\wp^{*}\right\|^{2} \\
& -\beta_{n}\left(1-\frac{\mu \xi_{n}}{\xi_{n+1}}\right)\left\|\varrho_{n}-v_{n}\right\|^{2}-\beta_{n}\left(1-\frac{\mu \xi_{n}}{\xi_{n+1}}\right)\left\|z_{n}-v_{n}\right\|^{2} .
\end{aligned}
$$

Because $\xi_{n} \rightarrow \xi$, then there exists a fixed number $\epsilon \in(0,1-\mu)$, such that

$$
\lim _{n \rightarrow \infty}\left(1-\frac{\mu \xi_{n}}{\xi_{n+1}}\right)=1-\mu>\epsilon>0
$$


Subsequently, there exist a fixed real number $N_{1} \in \mathcal{N}$ such that

$$
\left(1-\frac{\mu \xi_{n}}{\xi_{n+1}}\right)>\epsilon>0, \forall n \geq N_{1}
$$

Combining the expressions (20) and (21), we obtain

$$
\left\|u_{n+1}-\wp^{*}\right\|^{2} \leq\left\|\varrho_{n}-\wp^{*}\right\|^{2}, \forall n \geq N_{1} .
$$

By definition of the $\varrho_{n}$, we have

$$
\left\|\varrho_{n}-\wp^{*}\right\|=\left\|u_{n}+\theta_{n}\left(u_{n}-u_{n-1}\right)-\wp^{*}\right\| \leq\left\|u_{n}-\wp^{*}\right\|+\theta_{n}\left\|u_{n}-u_{n-1}\right\| .
$$

From the definition of $\varrho_{n}$ in Algorithm 1, we obtain

$$
\begin{aligned}
\left\|\varrho_{n}-\wp^{*}\right\|^{2} & =\left\|u_{n}+\theta_{n}\left(u_{n}-u_{n-1}\right)-\wp^{*}\right\|^{2} \\
& =\left\|\left(1+\theta_{n}\right)\left(u_{n}-\wp^{*}\right)-\theta_{n}\left(u_{n-1}-\wp^{*}\right)\right\|^{2} \\
& =\left(1+\theta_{n}\right)\left\|u_{n}-\wp^{*}\right\|^{2}-\theta_{n}\left\|u_{n-1}-\wp^{*}\right\|^{2}+\theta_{n}\left(1+\theta_{n}\right)\left\|u_{n}-u_{n-1}\right\|^{2} \\
& \leq\left(1+\theta_{n}\right)\left\|u_{n}-\wp^{*}\right\|^{2}-\theta_{n}\left\|u_{n-1}-\wp^{*}\right\|^{2}+2 \theta\left\|u_{n}-u_{n-1}\right\|^{2} .
\end{aligned}
$$

The expression (22) can also be written as

$$
\left\|u_{n+1}-\wp^{*}\right\| \leq\left\|u_{n}-\wp^{*}\right\|+\theta\left\|u_{n}-u_{n-1}\right\|, \quad \forall n \geq N_{1} .
$$

By using Lemma 4 with expressions (7) and (26), we have

$$
\lim _{n \rightarrow \infty}\left\|u_{n}-\wp^{*}\right\|=l, \text { for some finite } l \geq 0 .
$$

The equality (8) implies that

$$
\lim _{n \rightarrow \infty}\left\|u_{n}-u_{n-1}\right\|=0
$$

By letting $n \rightarrow \infty$ in (24) implies that

$$
\lim _{n \rightarrow \infty}\left\|\varrho_{n}-\wp^{*}\right\|=l .
$$

From the expression (20) and (25), we have

$$
\begin{aligned}
& \left\|u_{n+1}-\wp^{*}\right\|^{2} \\
& \leq\left(1+\theta_{n}\right)\left\|u_{n}-\wp^{*}\right\|^{2}-\theta_{n}\left\|u_{n-1}-\wp^{*}\right\|^{2}+2 \theta\left\|u_{n}-u_{n-1}\right\|^{2} \\
& \quad-\beta_{n}\left(1-\frac{\mu \xi_{n}}{\xi_{n+1}}\right)\left\|\varrho_{n}-v_{n}\right\|^{2}-\beta_{n}\left(1-\frac{\mu \xi_{n}}{\xi_{n+1}}\right)\left\|z_{n}-v_{n}\right\|^{2},
\end{aligned}
$$

which further implies that (for $n \geq N_{1}$ )

$$
\begin{aligned}
& \epsilon \beta\left\|\varrho_{n}-v_{n}\right\|^{2}+\epsilon \beta\left\|v_{n}-z_{n}\right\|^{2} \\
& \leq\left\|u_{n}-\wp^{*}\right\|^{2}-\left\|u_{n+1}-\wp^{*}\right\|^{2}+\theta_{n}\left(\left\|u_{n}-\wp^{*}\right\|^{2}-\left\|u_{n-1}-\wp^{*}\right\|^{2}\right)+2 \theta\left\|u_{n}-u_{n-1}\right\|^{2} .
\end{aligned}
$$

By letting $n \rightarrow \infty$ in (31), we obtain

$$
\lim _{n \rightarrow \infty}\left\|\varrho_{n}-v_{n}\right\|=\lim _{n \rightarrow \infty}\left\|v_{n}-z_{n}\right\|=0 .
$$


By using the Cauchy inequality and expression (32), we obtain

$$
\lim _{n \rightarrow \infty}\left\|\varrho_{n}-z_{n}\right\| \leq \lim _{n \rightarrow \infty}\left\|\varrho_{n}-v_{n}\right\|+\lim _{n \rightarrow \infty}\left\|z_{n}-v_{n}\right\|=0 .
$$

The expressions (29) and (32) imply that

$$
\lim _{n \rightarrow \infty}\left\|v_{n}-\wp^{*}\right\|=\lim _{n \rightarrow \infty}\left\|z_{n}-\wp^{*}\right\|=l .
$$

It follows from the expressions (27), (29) and (34) that the sequences $\left\{\varrho_{n}\right\},\left\{u_{n}\right\},\left\{v_{n}\right\}$ and $\left\{z_{n}\right\}$ are bounded. Now, we need to use Lemma 3 , for this it is compulsory to show that any weak sequential limit points of $\left\{u_{n}\right\}$ lies in the set $E P(f, \mathcal{K})$. Consider $z$ to be a weak limit point of $\left\{u_{n}\right\}$ i.e., there is a $\left\{u_{n_{k}}\right\}$ of $\left\{u_{n}\right\}$ that is weakly converges to $z$. Because $\left\|u_{n}-v_{n}\right\| \rightarrow 0$, then $\left\{v_{n_{k}}\right\}$ also weakly converge to $z$ and so $z \in \mathcal{K}$. Now, it is renaming to show that $z \in E P(f, \mathcal{K})$. From relation (11), due to $\xi_{n+1}$ and (17), we have

$$
\begin{aligned}
\xi_{n_{k}} f\left(v_{n_{k}}, y\right) \geq & \xi_{n_{k}} f\left(v_{n_{k}}, z_{n_{k}}\right)+\left\langle\varrho_{n_{k}}-z_{n_{k}}, y-z_{n_{k}}\right\rangle \\
\geq & \xi_{n_{k}} f\left(\varrho_{n_{k}}, z_{n_{k}}\right)-\xi_{n_{k}} f\left(\varrho_{n_{k}}, v_{n_{k}}\right)-\frac{\mu \xi_{n_{k}}}{2 \xi_{n_{k}+1}}\left\|\varrho_{n_{k}}-v_{n_{k}}\right\|^{2} \\
& -\frac{\mu \xi_{n_{k}}}{2 \xi_{n_{k}+1}}\left\|v_{n_{k}}-z_{n_{k}}\right\|^{2}+\left\langle\varrho_{n_{k}}-z_{n_{k}}, y-z_{n_{k}}\right\rangle \\
\geq & \left\langle\varrho_{n_{k}}-v_{n_{k}}, z_{n_{k}}-v_{n_{k}}\right\rangle-\frac{\mu \xi_{n_{k}}}{2 \xi_{n_{k}+1}}\left\|\varrho_{n_{k}}-v_{n_{k}}\right\|^{2} \\
& -\frac{\mu \xi_{n_{k}}}{2 \xi_{n_{k}+1}}\left\|v_{n_{k}}-z_{n_{k}}\right\|^{2}+\left\langle\varrho_{n_{k}}-z_{n_{k}} y-z_{n_{k}}\right\rangle,
\end{aligned}
$$

where $y \in \mathcal{K}$. It follows from (28), (32), (33) and the boundedness of $\left\{u_{n}\right\}$ right hand side tend to zero. Due to $\xi_{n_{k}}>0$, condition (f3) and $v_{n_{k}} \rightarrow z$, implies

$$
0 \leq \limsup _{k \rightarrow \infty} f\left(v_{n_{k}}, y\right) \leq f(z, y), \forall y \in \mathcal{K} .
$$

Because $z \in \mathcal{K}$ imply that $f(z, y) \geq 0, \forall y \in \mathcal{K}$. It is prove that $z \in E P(f, \mathcal{K})$. By Lemma 3, provides that $\left\{\varrho_{n}\right\},\left\{v_{n}\right\},\left\{z_{n}\right\}$ and $\left\{u_{n}\right\}$ weakly converges to $\wp^{*}$ as $n \rightarrow \infty$.

Finally, to prove that $\lim _{n \rightarrow \infty} P_{E P(f, \mathcal{K})}\left(u_{n}\right)=\wp^{*}$. Let $q_{n}:=P_{E P(f, \mathcal{K})}\left(u_{n}\right), \forall n \in \mathcal{N}$. For any $\wp^{*} \in E P(f, \mathcal{K})$, we have

$$
\left\|q_{n}\right\| \leq\left\|q_{n}-u_{n}\right\|+\left\|u_{n}\right\| \leq\left\|\wp^{*}-u_{n}\right\|+\left\|u_{n}\right\| .
$$

Clearly, the above implies that sequence $\left\{q_{n}\right\}$ is bounded. Next, we need to show that $\left\{q_{n}\right\}$ is a Cauchy sequence. By using Lemma 1(iii) and (23), we have

$$
\left\|u_{n+1}-q_{n+1}\right\| \leq\left\|u_{n+1}-q_{n}\right\| \leq\left\|u_{n}-q_{n}\right\|+\theta\left\|u_{n}-u_{n-1}\right\|, \quad \forall n \geq N_{1} .
$$

Thus, Lemma 4 provides the existence of $\lim _{n \rightarrow \infty}\left\|u_{n}-q_{n}\right\|$. Next, take (23) $\forall m>n \geq N_{1}$, we have

$$
\begin{aligned}
\left\|q_{n}-u_{m}\right\| & \leq\left\|q_{n}-u_{m-1}\right\|+\theta\left\|u_{n}-u_{n-1}\right\| \\
& \leq \cdots \leq\left\|q_{n}-u_{n}\right\|+\theta \sum_{k=n}^{m-1}\left\|u_{n}-u_{n-1}\right\| .
\end{aligned}
$$


Suppose that $q_{m}, q_{n} \in E P(f, \mathcal{K})$ for $m>n \geq N_{1}$, through Lemma 1(i) and (39), we have

$$
\begin{aligned}
& \left\|q_{n}-q_{m}\right\|^{2} \\
& \leq\left\|q_{n}-u_{m}\right\|^{2}-\left\|q_{m}-u_{m}\right\|^{2} \\
& \leq\left\|q_{n}-u_{n}\right\|^{2}+\left(\theta \sum_{k=n}^{m-1}\left\|u_{n}-u_{n-1}\right\|\right)^{2}+2 \theta\left\|q_{n}-u_{n}\right\| \sum_{k=n}^{m-1}\left\|u_{n}-u_{n-1}\right\|-\left\|q_{m}-u_{m}\right\|^{2} .
\end{aligned}
$$

The existence of $\lim _{n \rightarrow \infty}\left\|u_{n}-q_{n}\right\|$ and the summability of the series $\sum_{n}\left\|u_{n}-u_{n-1}\right\|<+\infty$, imply $\lim _{n \rightarrow \infty}\left\|q_{n}-q_{m}\right\|=0, \forall m>n$. As a result, $\left\{q_{n}\right\}$ is a Cauchy sequence and due the closeness of the set $E P(f, \mathcal{K})$ the sequence $\left\{q_{n}\right\}$ strongly converges to $q^{*} \in E P(f, \mathcal{K})$. Next, remaining to show that $q^{*}=\wp^{*}$. From Lemma 1(ii) and $\wp^{*}, q^{*} \in E P(f, \mathcal{K})$, we have

$$
\left\langle u_{n}-q_{n}, \wp^{*}-q_{n}\right\rangle \leq 0 .
$$

Because of $q_{n} \rightarrow q^{*}$ and $u_{n} \rightarrow \wp^{*}$, we obtain

$$
\left\langle\wp^{*}-q^{*}, \wp^{*}-q^{*}\right\rangle \leq 0,
$$

implies that $\wp^{*}=q^{*}=\lim _{n \rightarrow \infty} P_{E P(f, \mathcal{K})}\left(u_{n}\right)$.

\section{Applications to Solve Fixed Point Problems}

Now, consider the applications of our results that are discussed in Section 3 to solve fixed-point problems involving $\mathcal{K}$-strict pseudo-contraction. Let $T: \mathcal{K} \rightarrow \mathcal{K}$ be a mapping and the fixed point problem is formulated in the following manner:

$$
\text { Find } \wp^{*} \in \mathcal{K} \text { such as } T\left(\wp^{*}\right)=\wp^{*} .
$$

Let a mapping $T: \mathcal{K} \rightarrow \mathcal{K}$ is said to be

(i) sequentially weakly continuous on $\mathcal{K}$ if

$$
T\left(p_{n}\right) \rightarrow T(p) \text { for every sequence in } \mathcal{K} \text { satisfying } p_{n} \rightarrow p \text { (weakly converges); }
$$

(ii) $\quad \mathcal{K}$-strict pseudo-contraction [48] on $\mathcal{K}$ if

$$
\left\|T p_{1}-T p_{2}\right\|^{2} \leq\left\|p_{1}-p_{2}\right\|^{2}+\kappa\left\|\left(p_{1}-T p_{1}\right)-\left(p_{2}-T p_{2}\right)\right\|^{2}, \forall p_{1}, p_{2} \in \mathcal{K} ;
$$

that is equivalent to

$$
\left\langle T p_{1}-T p_{2}, p_{1}-p_{2}\right\rangle \leq\left\|p_{1}-p_{2}\right\|^{2}-\frac{1-\kappa}{2}\left\|\left(p_{1}-T p_{1}\right)-\left(p_{2}-T p_{2}\right)\right\|^{2}, \forall p_{1}, p_{2} \in \mathcal{K} .
$$


Note: if we define $f\left(p_{1}, p_{2}\right)=\left\langle p_{1}-T p_{1}, p_{2}-p_{1}\right\rangle, \forall p_{1}, p_{2} \in \mathcal{K}$. Then, the problem (1) convert into the fixed point problem with $2 k_{1}=2 k_{2}=\frac{3-2 \kappa}{1-\kappa}$. The value of $v_{n}$ in Algorithm 1 convert into followings:

$$
\begin{aligned}
v_{n} & =\underset{y \in \mathcal{K}}{\arg \min }\left\{\xi_{n} f\left(\varrho_{n}, y\right)+\frac{1}{2}\left\|\varrho_{n}-y\right\|^{2}\right\} \\
& =\underset{y \in \mathcal{K}}{\arg \min }\left\{\xi_{n}\left\langle\varrho_{n}-T\left(\varrho_{n}\right), y-\varrho_{n}\right\rangle+\frac{1}{2}\left\|\varrho_{n}-y\right\|^{2}\right\} \\
& =\underset{y \in \mathcal{K}}{\arg \min }\left\{\xi_{n}\left\langle\varrho_{n}-T\left(\varrho_{n}\right), y-\varrho_{n}\right\rangle+\frac{1}{2}\left\|\varrho_{n}-y\right\|^{2}+\frac{\xi_{n}^{2}}{2}\left\|\varrho_{n}-T\left(\varrho_{n}\right)\right\|^{2}-\frac{\xi_{n}^{2}}{2}\left\|\varrho_{n}-T\left(\varrho_{n}\right)\right\|^{2}\right\} \\
& =\underset{y \in \mathcal{K}}{\arg \min }\left\{\frac{1}{2}\left\|y-\varrho_{n}+\xi_{n}\left(\varrho_{n}-T\left(\varrho_{n}\right)\right)\right\|^{2}\right\} \\
& =P_{\mathcal{K}}\left[\varrho_{n}-\xi_{n}\left(\varrho_{n}-T\left(\varrho_{n}\right)\right)\right]=P_{\mathcal{K}}\left[\left(1-\xi_{n}\right) \varrho_{n}+\xi_{n} T\left(\varrho_{n}\right)\right] .
\end{aligned}
$$

In the similar way to the expression (44), we obtain

$$
z_{n}=P_{\mathcal{K}}\left[\varrho_{n}-\xi_{n}\left(v_{n}-T\left(v_{n}\right)\right)\right]
$$

As a consequence of the results in Section 3, we have the following fixed point theorem:

Corollary 1. Assume that $T: \mathcal{K} \rightarrow \mathcal{K}$ to be a weakly continuous and $\mathcal{K}$-strict pseudocontraction with Fix $(T) \neq \varnothing$. The sequences $\varrho_{n}, v_{n}, z_{n}$ and $u_{n}$ be generated in the following way:

(i) Choose $u_{-1}, u_{0} \in \mathcal{K}, \mu \in(0,1), \beta_{n} \in(0,1], \theta \in[0,1)$ and $\left\{\rho_{n}\right\} \subset[0,+\infty)$ satisfies the following condition:

$$
\sum_{n=0}^{+\infty} \rho_{n}<+\infty
$$

(ii) Choose $\theta_{n}$ satisfies $0 \leq \theta_{n} \leq \overline{\theta_{n}}$, such that

$$
\overline{\theta_{n}}= \begin{cases}\min \left\{\theta, \frac{\rho_{n}}{\left\|u_{n}-u_{n-1}\right\|}\right\} & \text { if } u_{n} \neq u_{n-1}, \\ \theta & \text { else. }\end{cases}
$$

(iii) Compute $u_{n+1}=\left(1-\beta_{n}\right) \varrho_{n}+\beta_{n} z_{n}$, where

$$
\left\{\begin{array}{l}
\varrho_{n}=u_{n}+\theta_{n}\left(u_{n}-u_{n-1}\right), \\
v_{n}=P_{\mathcal{K}}\left[\varrho_{n}-\xi_{n}\left(\varrho_{n}-T\left(\varrho_{n}\right)\right)\right] \\
z_{n}=P_{\mathcal{K}}\left[\varrho_{n}-\xi_{n}\left(v_{n}-T\left(v_{n}\right)\right)\right] .
\end{array}\right.
$$

(iv) Revised the stepsize $\xi_{n+1}$ in the following way:

$$
\xi_{n+1}=\min \left\{\xi_{n}, \frac{\mu\left\|\varrho_{n}-v_{n}\right\|^{2}+\mu\left\|z_{n}-v_{n}\right\|^{2}}{2\left[\left\langle\left(\varrho_{n}-v_{n}\right)-\left(T\left(\varrho_{n}\right)-T\left(v_{n}\right)\right), z_{n}-v_{n}\right\rangle\right]_{+}}\right\}
$$

Subsequently, $\left\{\varrho_{n}\right\},\left\{v_{n}\right\},\left\{z_{n}\right\}$ and $\left\{u_{n}\right\}$ be the sequences converges weakly to $\wp^{*} \in \operatorname{Fix}(T)$. 


\section{Application to Solve Variational Inequality Problems}

Now, consider the applications of our results that are discussed in Section 3 in order to solve variational inequality problems involving pseudomonotone and Lipschitz-type continuous operator. Let a operator $L: \mathcal{K} \rightarrow \mathcal{K}$ and the variational inequality problem is formulated as follows:

Find $\wp^{*} \in \mathcal{K}$ such that $\left\langle L\left(\wp^{*}\right), y-\wp^{*}\right\rangle \geq 0, \forall y \in \mathcal{K}$.

A mapping $L: \mathcal{E} \rightarrow \mathcal{E}$ is said to be

(i) L-Lipschitz continuous on $\mathcal{K}$ if

$$
\left\|L\left(p_{1}\right)-L\left(p_{2}\right)\right\| \leq L\left\|p_{1}-p_{2}\right\|, \forall p_{1}, p_{2} \in \mathcal{K}
$$

(ii) monotone on $\mathcal{K}$ if

$$
\left\langle L\left(p_{1}\right)-L\left(p_{2}\right), p_{1}-p_{2}\right\rangle \geq 0, \forall p_{1}, p_{2} \in \mathcal{K} ;
$$

(iii) pseudomonotone on $\mathcal{K}$ if

$$
\left\langle L\left(p_{1}\right), p_{2}-p_{1}\right\rangle \geq 0 \Longrightarrow\left\langle L\left(p_{2}\right), p_{1}-p_{2}\right\rangle \leq 0, \forall p_{1}, p_{2} \in \mathcal{K} .
$$

Note: let $f\left(p_{1}, p_{2}\right):=\left\langle L\left(p_{1}\right), p_{2}-p_{1}\right\rangle, \forall p_{1}, p_{2} \in \mathcal{K}$. Thus, problem (1) translates into the problem (VIP) with $L=2 k_{1}=2 k_{2}$. From the value of $v_{n}$, we have

$$
\begin{aligned}
v_{n} & =\underset{y \in \mathcal{K}}{\arg \min }\left\{\xi_{n} f\left(\varrho_{n}, y\right)+\frac{1}{2}\left\|\varrho_{n}-y\right\|^{2}\right\} \\
& =\underset{y \in \mathcal{K}}{\arg \min }\left\{\xi_{n}\left\langle L\left(\varrho_{n}\right), y-\varrho_{n}\right\rangle+\frac{1}{2}\left\|\varrho_{n}-y\right\|^{2}+\frac{\xi_{n}^{2}}{2}\left\|L\left(\varrho_{n}\right)\right\|^{2}-\frac{\xi_{n}^{2}}{2}\left\|L\left(\varrho_{n}\right)\right\|^{2}\right\} \\
& =\underset{y \in \mathcal{K}}{\arg \min }\left\{\frac{1}{2}\left\|y-\left(\varrho_{n}-\xi_{n} L\left(\varrho_{n}\right)\right)\right\|^{2}\right\} \\
& =P_{\mathcal{K}}\left[\varrho_{n}-\xi_{n} L\left(\varrho_{n}\right)\right] .
\end{aligned}
$$

In similar way to the expression (49), we obtain

$$
z_{n}=P_{\mathcal{K}}\left[\varrho_{n}-\xi_{n} L\left(v_{n}\right)\right] .
$$

Suppose that a mapping $L$ satisfies the following conditions:

(L1) $\quad L$ is monotone on $\mathcal{K}$ with $V I(L, \mathcal{K}) \neq \varnothing$;

(L2) $\quad L$ is $L$-Lipschitz continuous on $\mathcal{K}$ with $L>0$;

(L3) $\quad L$ is pseudomonotone on $\mathcal{K}$ with $V I(L, \mathcal{K}) \neq \varnothing$; and,

(L4) $\quad \limsup _{n \rightarrow \infty}\left\langle L\left(p_{n}\right), p-p_{n}\right\rangle \leq\langle L(p), y-p\rangle, \forall y \in \mathcal{K}$ and $\left\{p_{n}\right\} \subset \mathcal{K}$ satisfying $p_{n} \rightarrow p$.

Next, let $L$ to be monotone and (L4) can be removed. The condition (L4) is used to defined $f(u, v)=\langle L(u), v-u\rangle$ and satisfy the conditions (L4). The condition (f3) is required to show $z \in E P(f, \mathcal{K})$ see (36). The condition (L4) is required to show $z \in V I(L, \mathcal{K})$. Further, to show that $z \in V I(L, \mathcal{K})$. By letting the monotonicity of operator $L$, we have

$$
\left\langle L(y), y-v_{n}\right\rangle \geq\left\langle L\left(v_{n}\right), y-v_{n}\right\rangle, \forall y \in \mathcal{K} .
$$

By letting $f(u, v)=\langle L(u), v-u\rangle$ with expression (35), implies that

$$
\limsup _{k \rightarrow \infty}\left\langle L\left(v_{n_{k}}\right), y-v_{n_{k}}\right\rangle \geq 0, \forall y \in \mathcal{K} .
$$


Combining (50) with (51), we deduce that

$$
\limsup _{k \rightarrow \infty}\left\langle L(y), y-v_{n_{k}}\right\rangle \geq 0, \forall y \in \mathcal{K} .
$$

Therefore, $v_{n_{k}} \rightarrow z \in \mathcal{K}$, provides $\langle L(y), y-z\rangle \geq 0, \forall y \in \mathcal{K}$. Let $v_{t}=(1-t) z+t y, \forall t \in[0,1]$. Since $v_{t} \in \mathcal{K}$ for $t \in(0,1)$, we have

$$
0 \leq\left\langle L\left(v_{t}\right), v_{t}-z\right\rangle=t\left\langle L\left(v_{t}\right), y-z\right\rangle .
$$

That is $\left\langle L\left(v_{t}\right), y-z\right\rangle \geq 0$ every $t \in(0,1)$. Due to $v_{t} \rightarrow z$, while $t \rightarrow 0$, we have $\langle L(z), y-z\rangle \geq 0$, for all $y \in \mathcal{K}$, consequently $z \in V I(L, \mathcal{K})$.

Corollary 2. Let $L: \mathcal{K} \rightarrow \mathcal{E}$ be a mapping and satisfying the conditions (L1)-(L2). Assume that the sequences $\left\{\varrho_{n}\right\},\left\{v_{n}\right\},\left\{z_{n}\right\}$ and $\left\{u_{n}\right\}$ generated in the following manner:

(i) Choose $u_{-1}, u_{0} \in \mathcal{K}, \mu \in(0,1), \beta_{n} \in(0,1], \theta \in[0,1)$ and $\left\{\rho_{n}\right\} \subset[0,+\infty)$, such that

$$
\sum_{n=0}^{+\infty} \rho_{n}<+\infty
$$

(ii) Let $\theta_{n}$ satisfies $0 \leq \theta_{n} \leq \overline{\theta_{n}}$ and

$$
\overline{\theta_{n}}= \begin{cases}\min \left\{\theta, \frac{\rho_{n}}{\left\|u_{n}-u_{n-1}\right\|}\right\} & \text { if } u_{n} \neq u_{n-1}, \\ \theta & \text { otherwise. }\end{cases}
$$

(iii) Compute $u_{n+1}=\left(1-\beta_{n}\right) \varrho_{n}+\beta_{n} z_{n}$, where

$$
\left\{\begin{array}{l}
\varrho_{n}=u_{n}+\theta_{n}\left(u_{n}-u_{n-1}\right), \\
v_{n}=P_{\mathcal{K}}\left[\varrho_{n}-\xi_{n} L\left(\varrho_{n}\right)\right], \\
z_{n}=P_{\mathcal{K}}\left[\varrho_{n}-\xi_{n} L\left(v_{n}\right)\right] .
\end{array}\right.
$$

(iv) Stepsize $\xi_{n+1}$ is revised in the following way:

$$
\xi_{n+1}=\min \left\{\xi_{n}, \frac{\mu\left\|\varrho_{n}-v_{n}\right\|^{2}+\mu\left\|z_{n}-v_{n}\right\|^{2}}{2\left[\left\langle L\left(\varrho_{n}\right)-L\left(v_{n}\right), z_{n}-v_{n}\right\rangle\right]_{+}}\right\}
$$

Subsequently, the sequences $\left\{\varrho_{n}\right\},\left\{v_{n}\right\},\left\{z_{n}\right\}$ and $\left\{z_{n}\right\}$ converge weakly to $\wp^{*} \in V I(L, \mathcal{K})$.

Corollary 3. Let $L: \mathcal{K} \rightarrow \mathcal{E}$ be a mapping and satisfying the conditions (L2)-(L4). Assume that the sequences $\left\{\varrho_{n}\right\},\left\{v_{n}\right\},\left\{z_{n}\right\}$ and $\left\{u_{n}\right\}$ generated in the following manner:

(i) Choose $u_{-1}, u_{0} \in \mathcal{K}, \mu \in(0,1), \beta_{n} \in(0,1], \theta \in[0,1)$ and $\left\{\rho_{n}\right\} \subset[0,+\infty)$, such that

$$
\sum_{n=0}^{+\infty} \rho_{n}<+\infty
$$

(ii) Choose $\theta_{n}$ satisfying $0 \leq \theta_{n} \leq \overline{\theta_{n}}$, such that

$$
\overline{\theta_{n}}= \begin{cases}\min \left\{\theta, \frac{\rho_{n}}{\left\|u_{n}-u_{n-1}\right\|}\right\} & \text { if } u_{n} \neq u_{n-1}, \\ \theta & \text { else. }\end{cases}
$$


(iii) Compute $u_{n+1}=\left(1-\beta_{n}\right) \varrho_{n}+\beta_{n} z_{n}$, where

$$
\left\{\begin{array}{l}
\varrho_{n}=u_{n}+\theta_{n}\left(u_{n}-u_{n-1}\right), \\
v_{n}=P_{\mathcal{K}}\left[\varrho_{n}-\xi_{n} L\left(\varrho_{n}\right)\right] \\
z_{n}=P_{\mathcal{K}}\left[\varrho_{n}-\xi_{n} L\left(v_{n}\right)\right] .
\end{array}\right.
$$

(iv) The stepsize $\xi_{n+1}$ is updated in the following way:

$$
\xi_{n+1}=\min \left\{\xi_{n}, \frac{\mu\left\|\varrho_{n}-v_{n}\right\|^{2}+\mu\left\|z_{n}-v_{n}\right\|^{2}}{2\left[\left\langle L\left(\varrho_{n}\right)-L\left(v_{n}\right), z_{n}-v_{n}\right\rangle\right]_{+}}\right\}
$$

Subsequently, the sequences $\left\{\varrho_{n}\right\},\left\{v_{n}\right\},\left\{z_{n}\right\}$ and $\left\{z_{n}\right\}$ converge weakly to $\wp^{*} \in V I(L, \mathcal{K})$.

\section{Numerical Experiments}

The computational results present this section to prove the effectiveness of Algorithm 1 when compared to Algorithm 3.1 in [39] and Algorithm 1 in [38].

(i) For Algorithm 3.1 (Alg3.1) in [39]:

$$
\xi=\frac{1}{10 \max \left\{k_{1}, k_{2}\right\}}, \theta=\frac{1}{2}, \text { Error term }\left(D_{n}\right)=\max \left\{\left\|u_{n+1}-v_{n}\right\|^{2},\left\|u_{n+1}-\varrho_{n}\right\|^{2}\right\} .
$$

(ii) For Algorithm $1($ Alg1) in [38]:

$$
\xi=\frac{1}{4 \max \left\{k_{1}, k_{2}\right\}}, \theta=\frac{1}{2}, \rho_{n}=\frac{1}{n^{2}}, \text { Error term }\left(D_{n}\right)=\left\|\varrho_{n}-v_{n}\right\|^{2} .
$$

(iii) For Algorithm 1 (mAlg1):

$$
\xi=\frac{1}{2}, \theta=\frac{1}{2}, \mu=\frac{1}{3}, \rho_{n}=\frac{1}{n^{2}}, \beta_{n}=\frac{8}{10}, \text { Error term }\left(D_{n}\right)=\left\|\varrho_{n}-v_{n}\right\|^{2} .
$$

Example 1. Let take the Nash-Cournot Equilibrium Model that found in the paper [6]. A bifunction $f$ consider into the following form:

$$
f\left(p_{1}, p_{2}\right)=\left\langle P p_{1}+Q p_{2}+q, p_{2}-p_{1}\right\rangle,
$$

where $q \in \mathcal{R}^{m}$ with matrices $P, Q$ of order $m$ and Lipschitz constants are $k_{1}=k_{2}=\frac{1}{2}\|P-Q\|$ (see for more details [6]). In our case, $P, Q$ are taken at random (choose diagonal matrices $A_{1}$ and $A_{2}$ randomly entries from $[0,2]$ and $[-2,0]$, respectively. Two random orthogonal matrices $B_{1}$ and $B_{2}$ provide positive semidefinite matrix $M_{1}=B_{1} A_{1} B_{1}^{T}$ and negative semidefinite matrix $M_{2}=B_{2} A_{2} B_{2}^{T}$. Finally, set $Q=M_{1}+M_{1}^{T}, S=M_{2}+M_{2}^{T}$ and $P=Q-S$.) and elements of $q$ are taken arbitrary form $[-1,1]$. A set $\mathcal{K} \subset \mathcal{R}^{m}$ is taken as

$$
\mathcal{K}:=\left\{u \in \mathcal{R}^{m}:-10 \leq u_{i} \leq 10\right\} .
$$

Tables 1 and 2 and Figures $1-8$ presented the numerical results by taking $u_{-1}=u_{0}=v_{0}=(1, \cdots, 1)$ and $D_{n} \leq 10^{-9}$. 


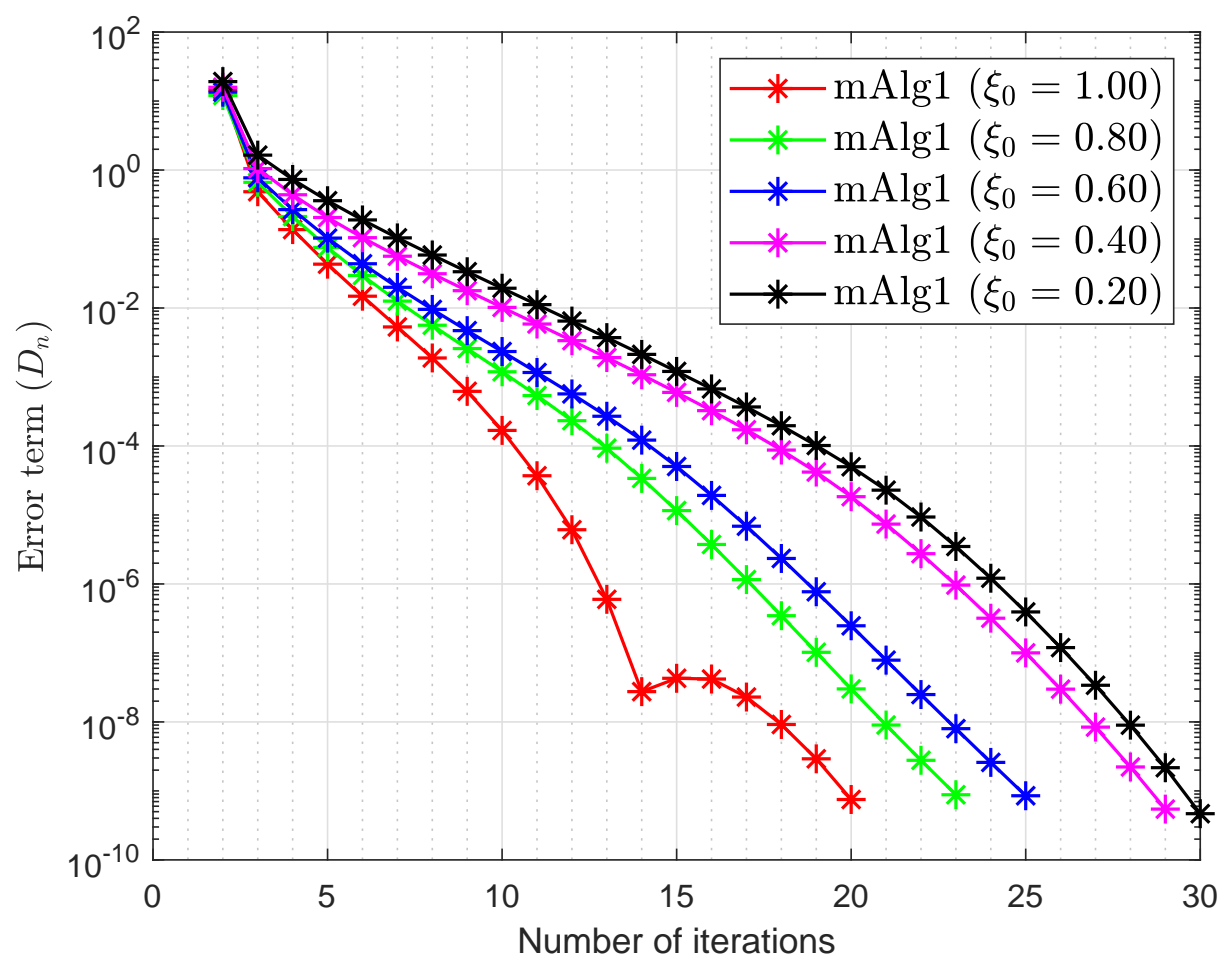

Figure 1. Example 1: numerical behaviour of Algorithm 1 by letting different options for $\xi_{0}$, while $\mathrm{m}=10$.

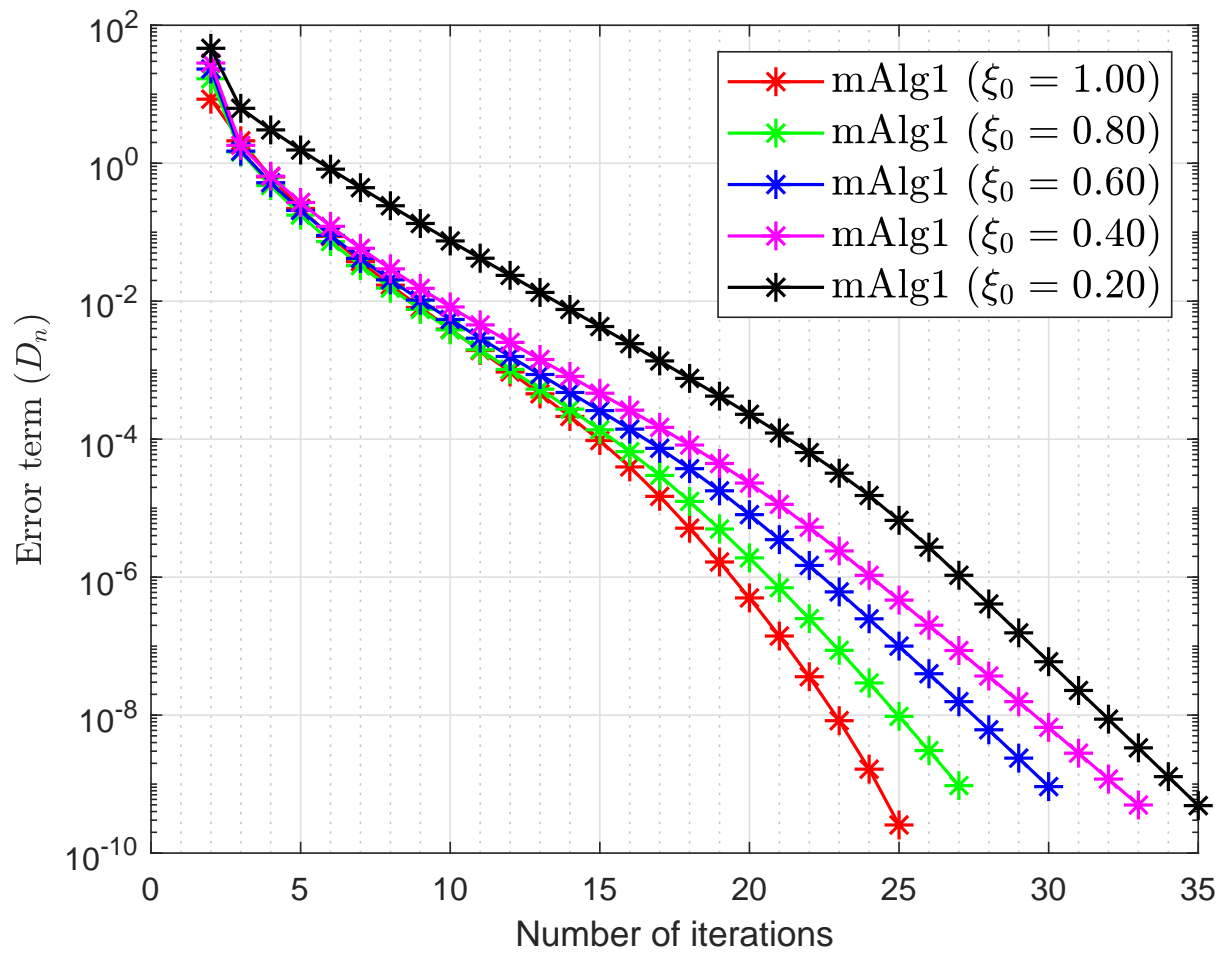

Figure 2. Example 1: numerical behaviour of Algorithm 1 by letting different options for $\xi_{0}$, while $\mathrm{m}=20$. 


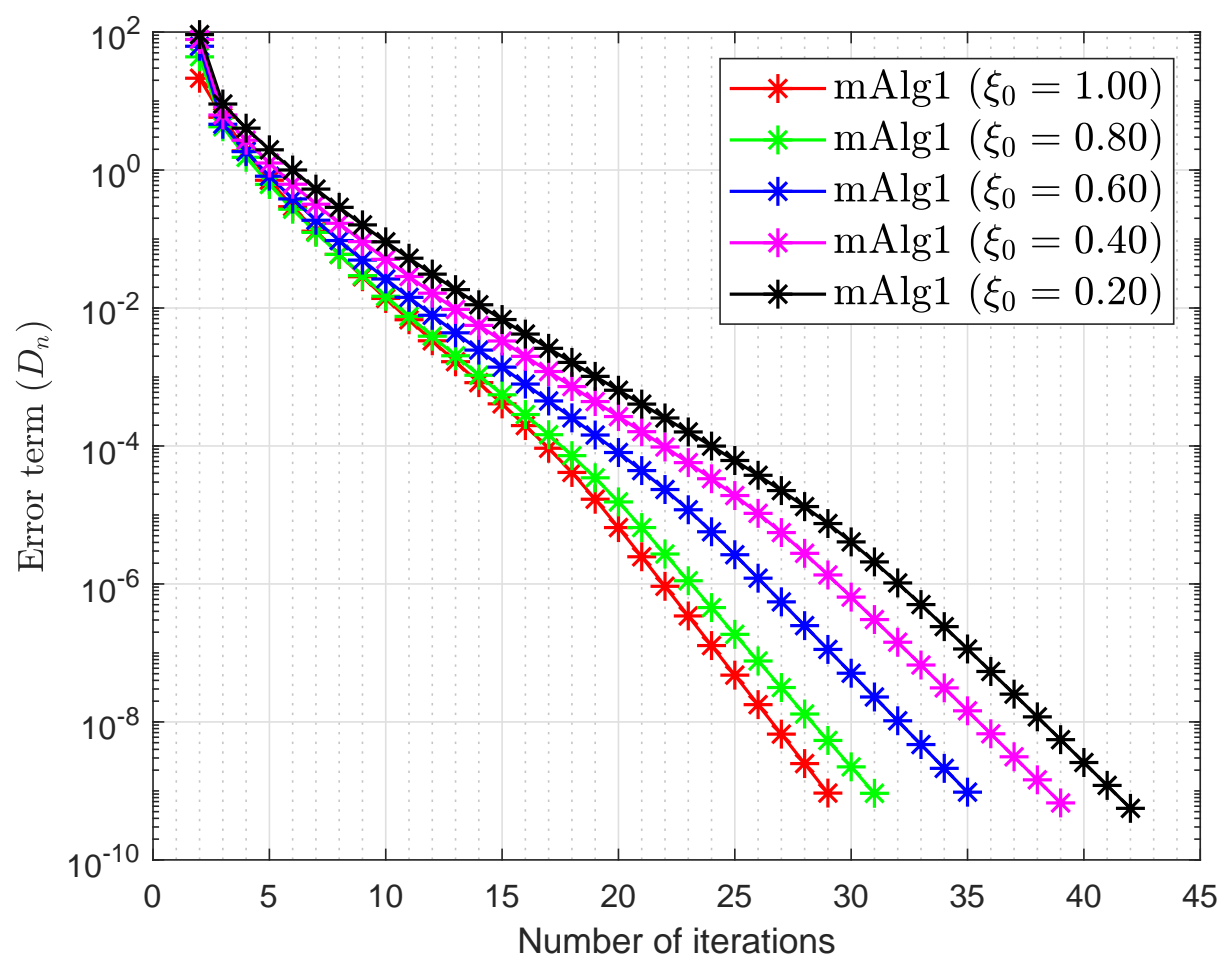

Figure 3. Example 1: numerical behaviour of Algorithm 1 by letting different options for $\xi_{0}$ while $\mathrm{m}=50$.

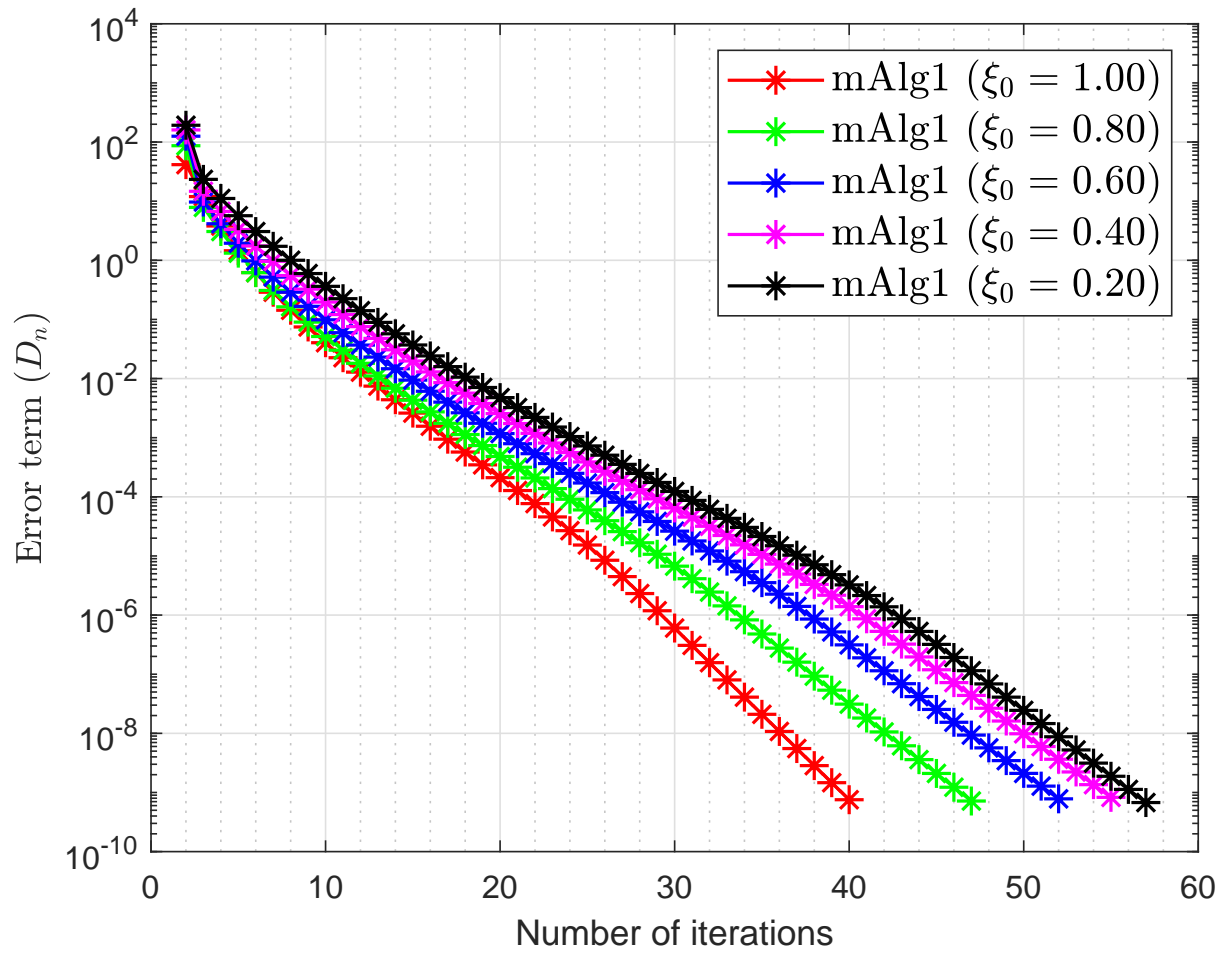

Figure 4. Example 1: numerical behaviour of Algorithm 1 by letting different options for $\xi_{0}$ while $\mathrm{m}=100$. 
Table 1. Example 1: Algorithm 1 numerical behaviour by letting different options for $\xi_{0}$ and $\mathrm{m}$.

\begin{tabular}{ccccccccc}
\hline & \multicolumn{2}{c}{$\mathbf{m}=\mathbf{1 0}$} & \multicolumn{2}{c}{$\mathbf{m}=\mathbf{2 0}$} & \multicolumn{2}{c}{$\mathbf{m}=\mathbf{5 0}$} & \multicolumn{2}{c}{$\mathbf{m}=\mathbf{1 0 0}$} \\
\hline $\boldsymbol{\xi}_{\mathbf{0}}$ & iter. & time & iter. & time & iter. & time & iter. & time \\
\hline 1.00 & 20 & 0.1701 & 25 & 0.2153 & 29 & 0.2726 & 40 & 0.5570 \\
0.80 & 23 & 0.1945 & 27 & 0.2326 & 31 & 0.2788 & 47 & 0.5469 \\
0.60 & 25 & 0.1995 & 30 & 0.2634 & 35 & 0.3285 & 52 & 0.6228 \\
0.40 & 29 & 0.1467 & 33 & 0.2979 & 39 & 0.3549 & 55 & 0.6542 \\
0.20 & 30 & 0.2632 & 35 & 0.2868 & 42 & 0.3849 & 57 & 0.6662 \\
\hline
\end{tabular}
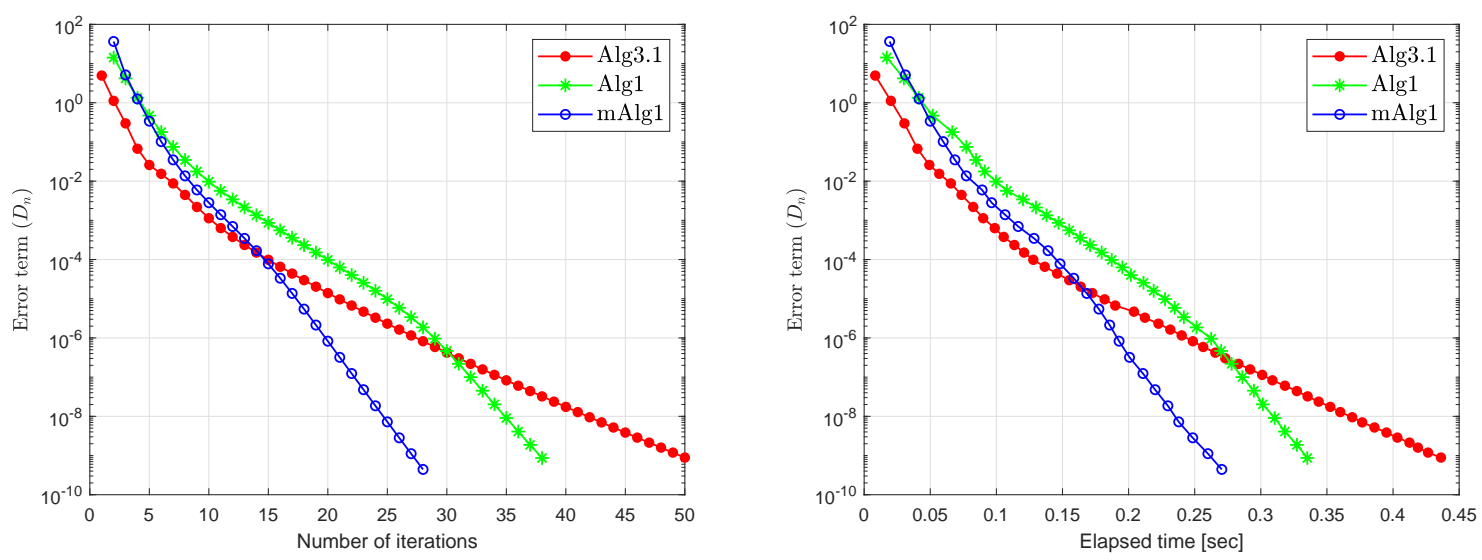

Figure 5. Example 1: Algorithm 1 (mAlg1) numerical comparison with Algorithm 3.1 (Alg3.1) in [39] and Algorithm $1(\mathrm{Alg} 1)$ in [38] while $\mathrm{m}=60$.
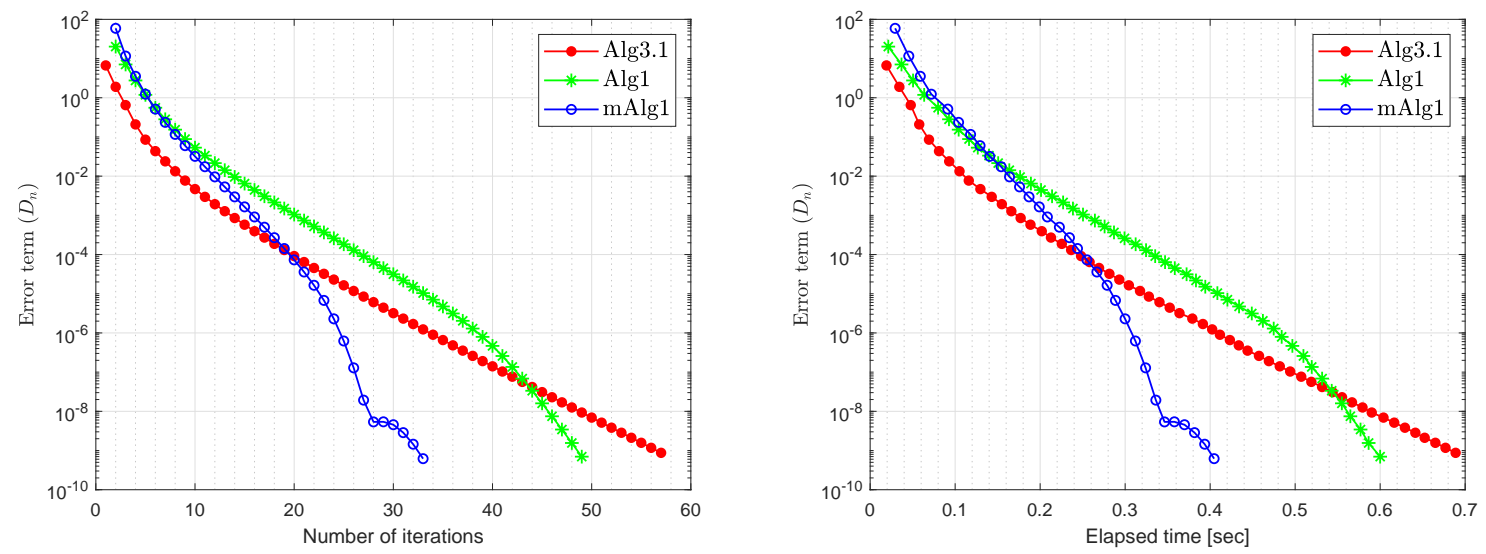

Figure 6. Example 1: Algorithm 1 (mAlg1) numerical comparison with Algorithm 3.1 (Alg3.1) in [39] and Algorithm 1 (Alg1) in [38] while $\mathrm{m}=120$. 

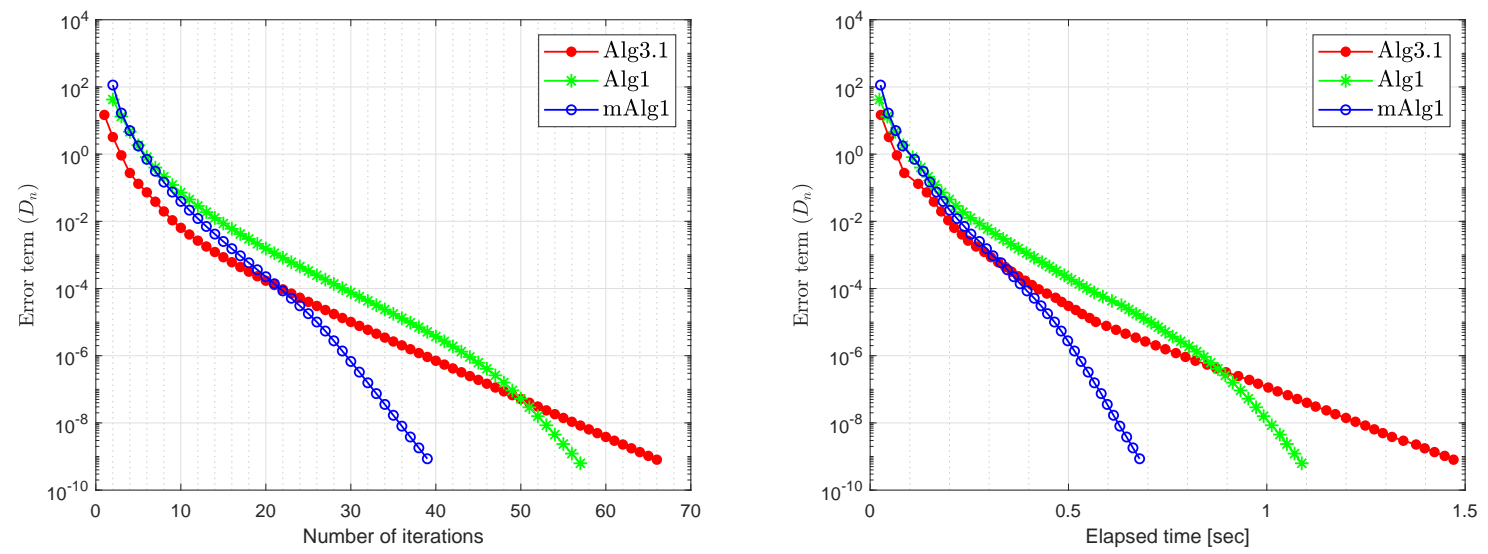

Figure 7. Example 1: Algorithm 1 (mAlg1) numerical comparison with Algorithm 3.1 (Alg3.1) in [39] and Algorithm 1 (Alg1) in [38] while $\mathrm{m}=200$.
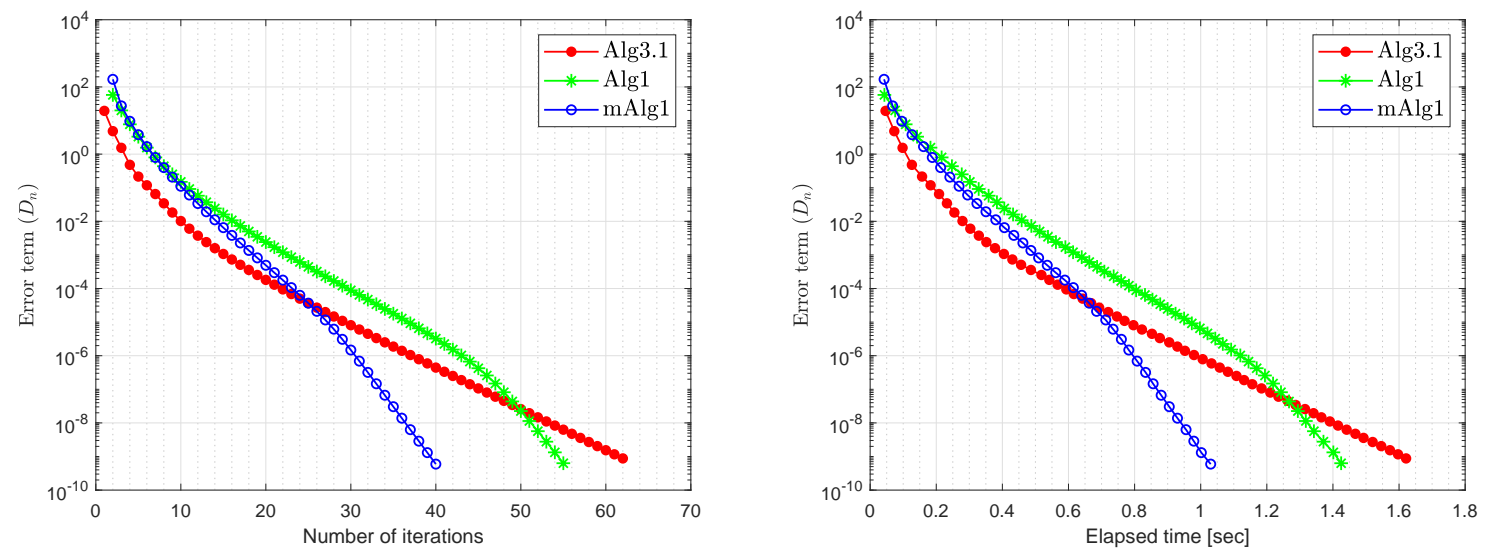

Figure 8. Example 1: Algorithm 1 (mAlg1) numerical comparison with Algorithm 3.1 (Alg3.1) in [39] and Algorithm 1 (Alg1) in [38] while $\mathrm{m}=300$.

Table 2. Example 1: Algorithm 1 (mAlg1) numerical comparison with Algorithm 3.1 (Alg3.1) in [39] and Algorithm 1 (Alg1) in [38].

\begin{tabular}{ccccccc}
\hline & \multicolumn{3}{c}{ Number of Iterations } & \multicolumn{3}{c}{ Execution Time in Seconds } \\
\hline $\boldsymbol{m}$ & Alg3.1 & Alg1 & mAlg1 & Alg3.1 & Alg1 & mAlg1 \\
\hline 60 & 50 & 38 & 28 & 0.4362 & 0.3352 & 0.2705 \\
120 & 57 & 49 & 33 & 0.6888 & 0.6000 & 0.4047 \\
200 & 66 & 57 & 39 & 1.4708 & 1.0881 & 0.6794 \\
300 & 62 & 55 & 40 & 1.6213 & 1.4251 & 1.0303 \\
\hline
\end{tabular}

Example 2. Suppose that $f: \mathcal{K} \times \mathcal{K} \rightarrow \mathcal{R}$ be a bifunction defined in the following way

$$
f(p, q)=\sum_{i=2}^{5}\left(q_{i}-p_{i}\right)\|p\|, \forall p, q \in \mathcal{R}^{5}
$$

where $\mathcal{K}=\left\{\left(p_{1}, \cdots, p_{5}\right): p_{1} \geq-1, p_{i} \geq 1, i=2, \cdots, 5\right\}$. A bifunction $f$ is Lipschitz-type continuous with constants $k_{1}=k_{2}=2$ and satisfy the conditions (f1)-(f4). In order to evaluate the best possible value of the control parameters, a numerical test is performed taking the variation of the inertial factor $\theta$. The numerical comparison results are shown in the Table 3 by using $u_{-1}=u_{0}=v_{0}=(2,3,2,5,5)$ and $D_{n} \leq 10^{-6}$. 
Table 3. Example 2: Algorithm 1 (mAlg1) numerical comparison with Algorithm 3.1 (Alg3.1) in [39] and Algorithm 1 (Alg1) in [38].

\begin{tabular}{ccccccc}
\hline & \multicolumn{3}{c}{ Number of Iterations } & \multicolumn{3}{c}{ Execution Time in Seconds } \\
\hline $\boldsymbol{\theta}$ & Alg3.1 & Alg1 & mAlg1 & Alg3.1 & Alg1 & mAlg1 \\
\hline 0.90 & 67 & 56 & 47 & 2.8674 & 2.5324 & 1.6734 \\
0.70 & 63 & 53 & 45 & 2.7813 & 2.6423 & 1.5026 \\
0.50 & 57 & 47 & 41 & 2.0912 & 2.4212 & 1.4991 \\
0.30 & 61 & 48 & 44 & 2.4115 & 2.3567 & 1.5092 \\
0.10 & 69 & 60 & 47 & 2.9229 & 2.2881 & 1.5098 \\
\hline
\end{tabular}

Example 3. Let $\mathcal{E}=L^{2}([0,1])$ be a Hilbert space with an inner product $\langle p, q\rangle=\int_{0}^{1} p(r) q(r) d r$, and the induced norm $\|p\|=\sqrt{\int_{0}^{1} p^{2}(r) d r}, \forall p, q \in \mathcal{E}$. The set $\mathcal{K}:=\left\{p \in L^{2}([0,1]): \int_{0}^{1} r p(r) d r=2\right\}$. Suppose that $f: \mathcal{E} \times \mathcal{E} \rightarrow \mathcal{R}$ is defined by

$$
f(p, q)=\langle L(p), q-p\rangle
$$

where $L(p(r))=\int_{0}^{r} p(s) d s$, for every $p \in L^{2}([0,1])$ and $r \in[0,1]$. The projection on set $\mathcal{K}$ is computed in the following way:

$$
P_{\mathcal{K}}(p)(r):=p(r)-\frac{\int_{0}^{1} r p(r) d r-2}{\int_{0}^{1} r^{2} d r} r, r \in[0,1] .
$$

Table 4 reports the numerical results by using stopping criterion $D_{n} \leq 10^{-6}$ and letting $u_{-1}=u_{0}=v_{0}$.

Table 4. Example 3: Algorithm 1 (mAlg1) numerical comparison with Algorithm 3.1 (Alg3.1) in [39] and Algorithm 1 (Alg1) in [38].

\begin{tabular}{ccccccc}
\hline & \multicolumn{3}{c}{ Number of Iterations } & \multicolumn{3}{c}{ Execution time in Seconds } \\
\hline $\boldsymbol{u}_{\mathbf{0}}$ & Alg3.1 & Alg1 & mAlg1 & Alg3.1 & Alg1 & mAlg1 \\
\hline $3 t$ & 33 & 28 & 19 & 4.7654 & 3.9782 & 2.9342 \\
$3 t^{2}$ & 38 & 31 & 20 & 5.2598 & 4.1458 & 3.0987 \\
$3 \sin (t)$ & 41 & 33 & 22 & 5.9876 & 5.3976 & 4.4298 \\
$3 \cos (t)$ & 47 & 39 & 22 & 6.9921 & 5.4765 & 4.4611 \\
$3 \exp (t)^{2}$ & 58 & 43 & 31 & 8.4691 & 5.8329 & 5.0321 \\
\hline
\end{tabular}

Example 4. Assume that a bifunction $f$ is defined by

$$
f(p, q)=\langle L(p), q-p\rangle \text { and } L(p)=G(p)+H(p)
$$

where

$$
G(p)=\left(g_{1}(p), g_{2}(p), \cdots, g_{m}(p)\right), H(p)=E p+c, c=(-1,-1, \cdots,-1),
$$

and

$$
g_{i}(p)=p_{i-1}^{2}+p_{i}^{2}+p_{i-1} p_{i}+p_{i} p_{i+1}, \quad i=1,2, \ldots, m, \quad p_{0}=p_{m+1}=0 .
$$

Let the matrix E of order $m$ are consider in the following way:

$$
e_{i, j}= \begin{cases}4 & j=i \\ 1 & i-j=1 \\ -2 & i-j=-1 \\ 0 & \text { otherwise }\end{cases}
$$


where $\mathcal{K}=\left\{\left(u_{1}, \cdots, u_{m}\right) \in \mathcal{R}^{m}: u_{i} \geq 1, i=2, \cdots, m\right\}$. Figures 9-13 and Table 5 report the numerical results by taking $u_{-1}=u_{0}=v_{0}=(1, \cdots, 1)$ and $D_{n} \leq 10^{-6}$.
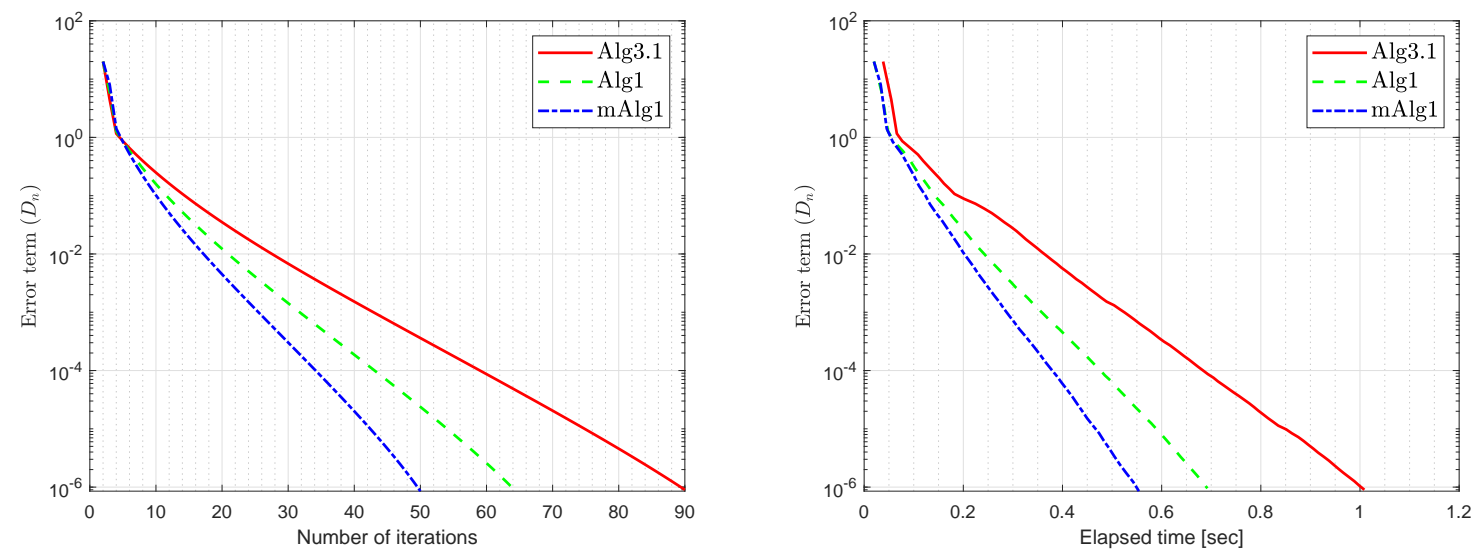

Figure 9. Example 4: Algorithm 1 (mAlg1) numerical comparison with Algorithm 3.1 (Alg3.1) in [39] and Algorithm $1(\mathrm{Alg} 1)$ in [38] while $\mathrm{m}=20$.
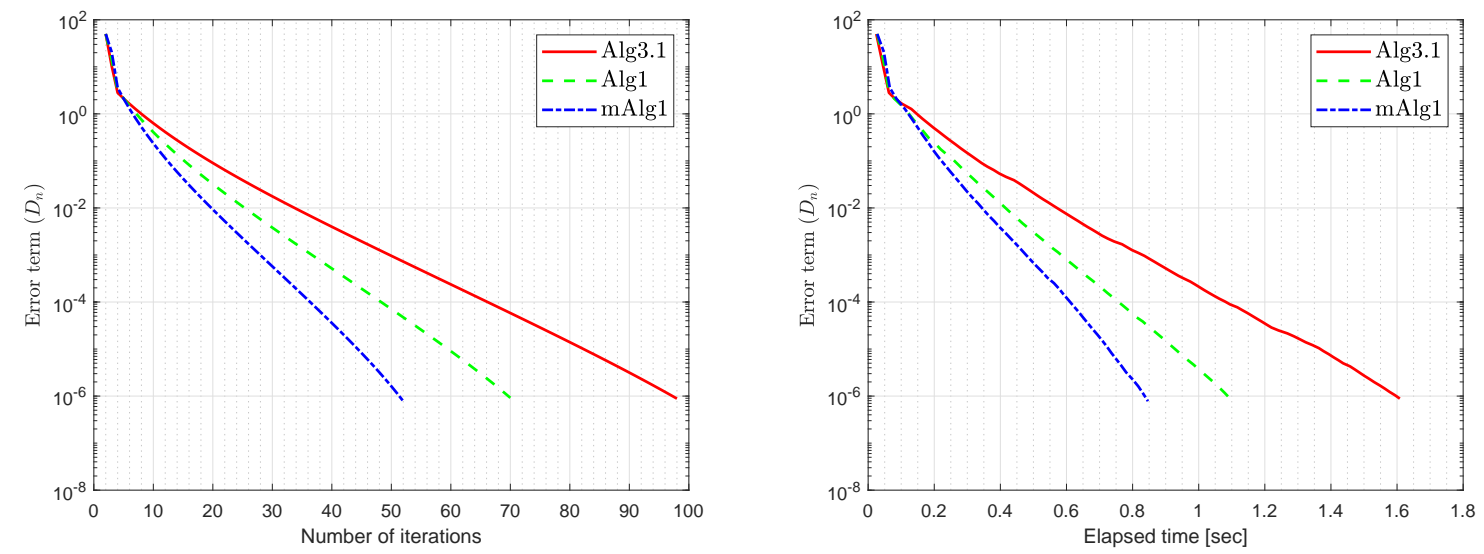

Figure 10. Example 4: Algorithm 1 (mAlg1) numerical comparison with Algorithm 3.1 (Alg3.1) in [39] and Algorithm 1 (Alg1) in [38] while $\mathrm{m}=50$.
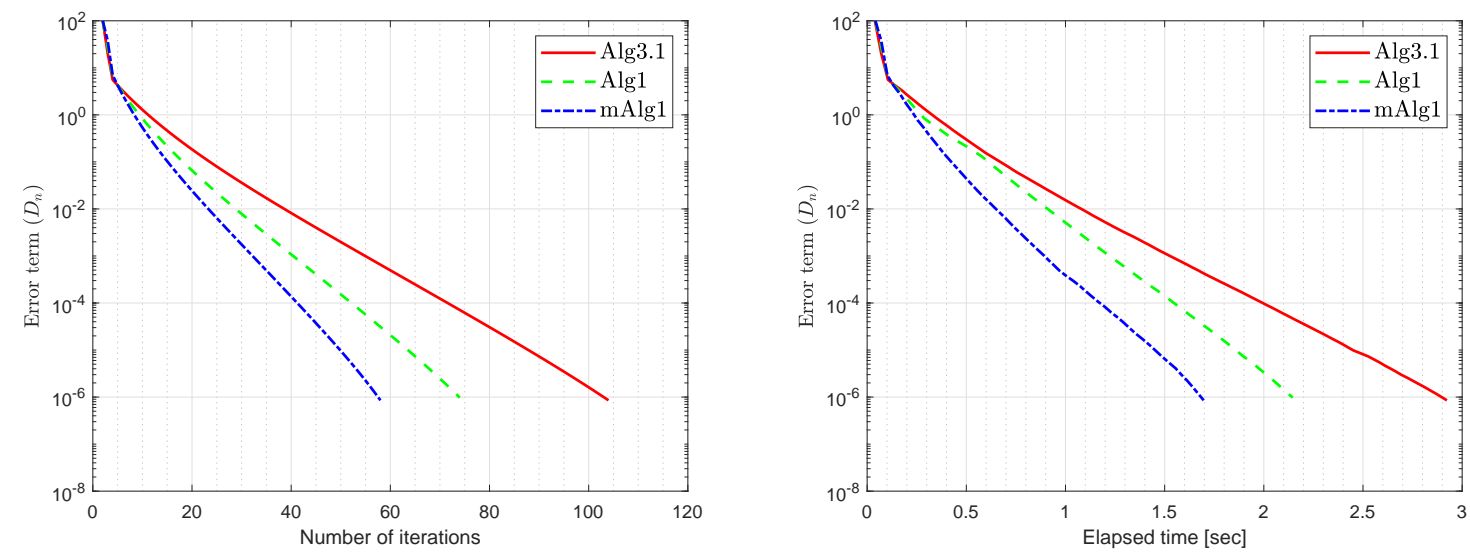

Figure 11. Example 4: Algorithm 1 (mAlg1) numerical comparison with Algorithm 3.1 (Alg3.1) in [39] and Algorithm 1 (Alg1) in [38] while $\mathrm{m}=100$. 

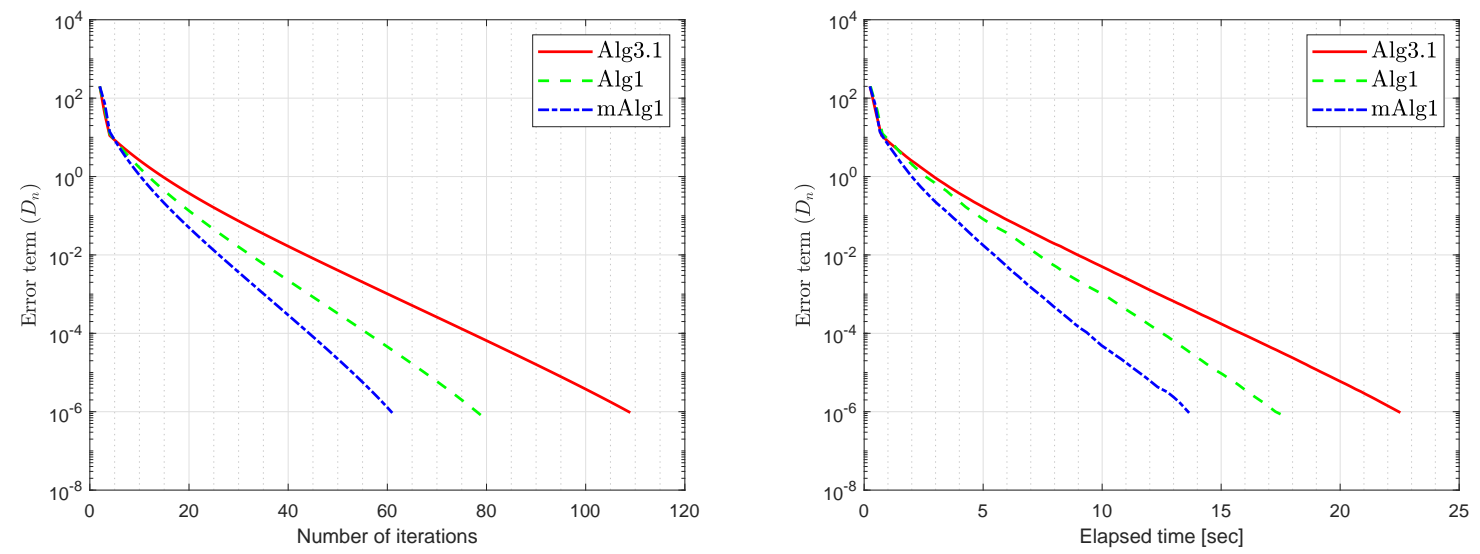

Figure 12. Example 4: Algorithm 1 (mAlg1) numerical comparison with Algorithm 3.1 (Alg3.1) in [39] and Algorithm 1 (Alg1) in [38] while $\mathrm{m}=200$.
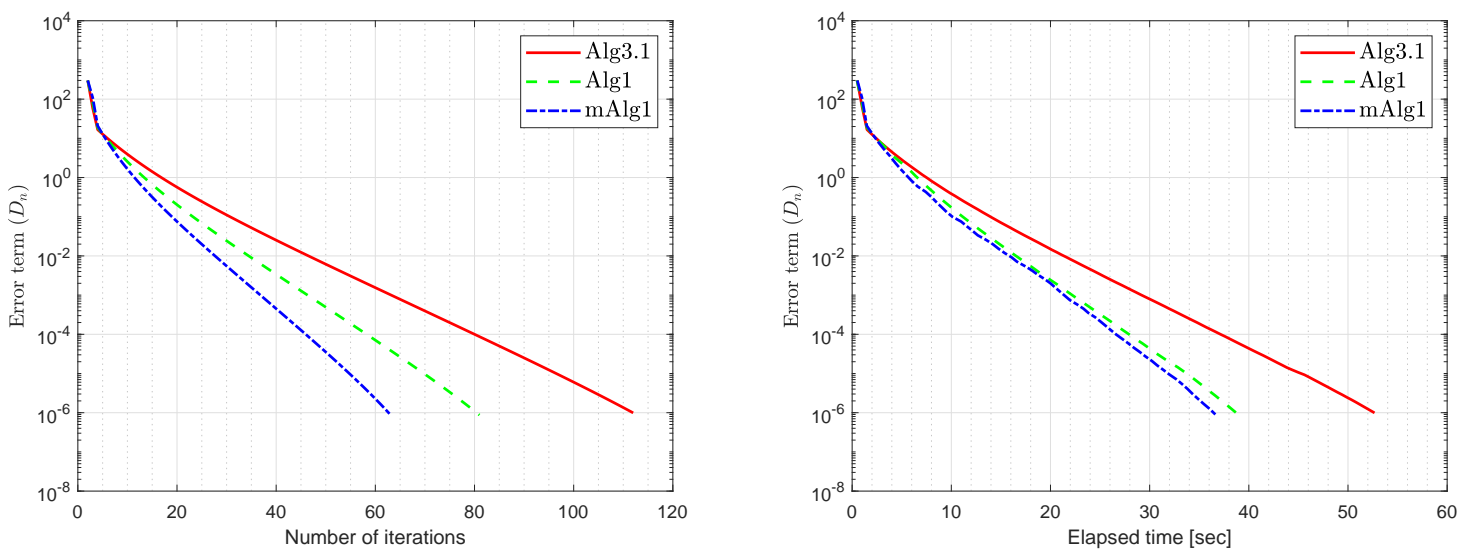

Figure 13. Example 4: Algorithm 1 (mAlg1) numerical comparison with Algorithm 3.1 (Alg3.1) in [39] and Algorithm 1 (Alg1) in [38] while $\mathrm{m}=300$.

Table 5. Example 4: Algorithm 1 (mAlg1) numerical comparison with Algorithm 3.1 (Alg3.1) in [39] and Algorithm 1 (Alg1) in [38].

\begin{tabular}{ccccccc}
\hline & \multicolumn{3}{c}{ Number of Iterations } & \multicolumn{3}{c}{ Execution Time in Seconds } \\
\hline $\boldsymbol{m}$ & Alg3.1 & Alg1 & mAlg1 & Alg3.1 & Alg1 & mAlg1 \\
\hline 20 & 90 & 64 & 50 & 1.0089 & 0.6923 & 0.5541 \\
50 & 98 & 70 & 52 & 1.6089 & 1.9092 & 0.8464 \\
100 & 104 & 74 & 58 & 2.9231 & 2.1456 & 1.6970 \\
200 & 109 & 79 & 61 & 22.5299 & 17.6267 & 13.6542 \\
300 & 112 & 81 & 63 & 52.6776 & 39.0018 & 36.6305 \\
\hline
\end{tabular}

\section{Remark 2.}

(i) It is also significant that the value of $\xi_{0}$ is crucial and performs best when it is nearer to 1 .

(ii) It is observed that the selection of the value $\vartheta$ is often significant and roughly the value $\vartheta \in(3,6)$ performs better than most other values.

\section{Conclusions}

In this paper, we consider the convergence result for pseudomonotone equilibrium problems that involve Lipschitz-type continuous bifunction but the Lipschitz-type constants are unknown. We modify the extragradient methods with an inertial term and new step size formula. Weak convergence theorem 
is proved for sequences generated by the algorithm. Several numerical experiments confirm the effectiveness of the proposed algorithms.

Author Contributions: Conceptualization, H.u.R., N.P. and M.D.1.S.; Writing-Original Draft Preparation, N.W., N.P. and H.u.R.; Writing-Review \& Editing, N.W., N.P., H.u.R. and M.D.l.S.; Methodology, N.P. and H.u.R.; Visualization, N.W. and N.P.; Software, H.u.R.; Funding Acquisition, M.D.l.S.; Supervision, M.D.l.S. and H.u.R.; Project Administration; M.D.1.S.; Resources; M.D.I.S. and H.u.R. All authors have read and agreed to the published version of this manuscript.

Funding: This research work was financially supported by Spanish Government for Grant RTI2018-094336-B-I00 (MCIU/AEI/FEDER, UE) and to the Basque Government for Grant IT1207-19.

Acknowledgments: We are very grateful to the Editor and the anonymous referees for their valuable and useful comments, which helped improve the quality of this work. Nopparat Wairojjana was partially supported by Valaya Alongkorn Rajabhat University under the Royal Patronage, Thailand. The corresponding author are grateful to the Spanish Government for Grant RTI2018-094336-B-I00 (MCIU/AEI/FEDER, UE) and to the Basque Government for Grant IT1207-19.

Conflicts of Interest: The authors declare no conflict of interest.

\section{References}

1. Blum, E. From optimization and variational inequalities to equilibrium problems. Math. Stud. 1994, 63, 123-145.

2. Fan, K. A Minimax Inequality and Applications, Inequalities III; Shisha, O., Ed.; Academic Press: New York, NY, USA, 1972.

3. Facchinei, F.; Pang, J.S. Finite-Dimensional Variational Inequalities and Complementarity Problems; Springer Science \& Business Media: Berlin, Germany, 2007.

4. Konnov, I. Equilibrium Models and Variational Inequalities; Elsevier: Amsterdam, The Netherlands, 2007; Volume 210.

5. Muu, L.D.; Oettli, W. Convergence of an adaptive penalty scheme for finding constrained equilibria. Nonlinear Anal. Theory Methods Appl. 1992, 18, 1159-1166. [CrossRef]

6. Quoc, T.D.; Le Dung, M.N.V.H. Extragradient algorithms extended to equilibrium problems. Optimization 2008, 57, 749-776. [CrossRef]

7. Quoc, T.D.; Anh, P.N.; Muu, L.D. Dual extragradient algorithms extended to equilibrium problems. J. Glob. Optim. 2011, 52, 139-159. [CrossRef]

8. Lyashko, S.I.; Semenov, V.V. A New Two-Step Proximal Algorithm of Solving the Problem of Equilibrium Programming. In Optimization and Its Applications in Control and Data Sciences; Springer International Publishing: Berlin/Heidelberg, Germany, 2016; pp. 315-325. [CrossRef]

9. Takahashi, S.; Takahashi, W. Viscosity approximation methods for equilibrium problems and fixed point problems in Hilbert spaces. J. Math. Anal. Appl. 2007, 331, 506-515. [CrossRef]

10. ur Rehman, H.; Kumam, P.; Cho, Y.J.; Yordsorn, P. Weak convergence of explicit extragradient algorithms for solving equilibirum problems. J. Inequalities Appl. 2019, 2019. [CrossRef]

11. Anh, P.N.; Hai, T.N.; Tuan, P.M. On ergodic algorithms for equilibrium problems. J. Glob. Optim. 2015, 64, 179-195. [CrossRef]

12. Hieu, D.V.; Quy, P.K.; Vy, L.V. Explicit iterative algorithms for solving equilibrium problems. Calcolo $2019,56$. [CrossRef]

13. Hieu, D.V. New extragradient method for a class of equilibrium problems in Hilbert spaces. Appl. Anal. 2017, 97, 811-824. [CrossRef]

14. ur Rehman, H.; Kumam, P.; Je Cho, Y.; Suleiman, Y.I.; Kumam, W. Modified Popov's explicit iterative algorithms for solving pseudomonotone equilibrium problems. Optim. Methods Softw. 2020, pp. 1-32. [CrossRef]

15. ur Rehman, H.; Kumam, P.; Abubakar, A.B.; Cho, Y.J. The extragradient algorithm with inertial effects extended to equilibrium problems. Comput. Appl. Math. 2020, 39. [CrossRef]

16. Santos, P.; Scheimberg, S. An inexact subgradient algorithm for equilibrium problems. Comput. Appl. Math. 2011, 30, 91-107. 
17. Hieu, D.V. Halpern subgradient extragradient method extended to equilibrium problems. Revista de la Real Academia de Ciencias Exactas, Físicas y Naturales Serie A Matemáticas 2016, 111, 823-840. [CrossRef]

18. ur Rehman, H.; Kumam, P.; Kumam, W.; Shutaywi, M.; Jirakitpuwapat, W. The Inertial Sub-Gradient Extra-Gradient Method for a Class of Pseudo-Monotone Equilibrium Problems. Symmetry 2020, 12, 463. [CrossRef]

19. Anh, P.N.; An, L.T.H. The subgradient extragradient method extended to equilibrium problems. Optimization 2012, 64, 225-248. [CrossRef]

20. Muu, L.D.; Quoc, T.D. Regularization Algorithms for Solving Monotone Ky Fan Inequalities with Application to a Nash-Cournot Equilibrium Model. J. Optim. Theory Appl. 2009, 142, 185-204. [CrossRef]

21. ur Rehman, H.; Kumam, P.; Argyros, I.K.; Deebani, W.; Kumam, W. Inertial Extra-Gradient Method for Solving a Family of Strongly Pseudomonotone Equilibrium Problems in Real Hilbert Spaces with Application in Variational Inequality Problem. Symmetry 2020, 12, 503. [CrossRef]

22. ur Rehman, H.; Kumam, P.; Argyros, I.K.; Alreshidi, N.A.; Kumam, W.; Jirakitpuwapat, W. A Self-Adaptive Extra-Gradient Methods for a Family of Pseudomonotone Equilibrium Programming with Application in Different Classes of Variational Inequality Problems. Symmetry 2020, 12, 523. [CrossRef]

23. ur Rehman, H.; Kumam, P.; Argyros, I.K.; Shutaywi, M.; Shah, Z. Optimization Based Methods for Solving the Equilibrium Problems with Applications in Variational Inequality Problems and Solution of Nash Equilibrium Models. Mathematics 2020, 8, 822. [CrossRef]

24. Yordsorn, P.; Kumam, P.; ur Rehman, H.; Ibrahim, A.H. A Weak Convergence Self-Adaptive Method for Solving Pseudomonotone Equilibrium Problems in a Real Hilbert Space. Mathematics 2020, 8, 1165. [CrossRef]

25. Yordsorn, P.; Kumam, P.; Rehman, H.U. Modified two-step extragradient method for solving the pseudomonotone equilibrium programming in a real Hilbert space. Carpathian J. Math. 2020, 36, 313-330.

26. La Sen, M.D.; Agarwal, R.P.; Ibeas, A.; Alonso-Quesada, S. On the Existence of Equilibrium Points, Boundedness, Oscillating Behavior and Positivity of a SVEIRS Epidemic Model under Constant and Impulsive Vaccination. Adv. Differ. Equ. 2011, 2011, 1-32. [CrossRef]

27. La Sen, M.D.; Agarwal, R.P. Some fixed point-type results for a class of extended cyclic self-mappings with a more general contractive condition. Fixed Point Theory Appl. 2011, 2011. [CrossRef]

28. Wairojjana, N.; ur Rehman, H.; Argyros, I.K.; Pakkaranang, N. An Accelerated Extragradient Method for Solving Pseudomonotone Equilibrium Problems with Applications. Axioms 2020, 9, 99. [CrossRef]

29. La Sen, M.D. On Best Proximity Point Theorems and Fixed Point Theorems for -Cyclic Hybrid Self-Mappings in Banach Spaces. Abstr. Appl. Anal. 2013, 2013, 1-14. [CrossRef]

30. ur Rehman, H.; Kumam, P.; Shutaywi, M.; Alreshidi, N.A.; Kumam, W. Inertial Optimization Based Two-Step Methods for Solving Equilibrium Problems with Applications in Variational Inequality Problems and Growth Control Equilibrium Models. Energies 2020, 13, 3292. [CrossRef]

31. Rehman, H.U.; Kumam, P.; Dong, Q.L.; Peng, Y.; Deebani, W. A new Popov's subgradient extragradient method for two classes of equilibrium programming in a real Hilbert space. Optimization 2020, 1-36. [CrossRef]

32. Wang, L.; Yu, L.; Li, T. Parallel extragradient algorithms for a family of pseudomonotone equilibrium problems and fixed point problems of nonself-nonexpansive mappings in Hilbert space. J. Nonlinear Funct. Anal. 2020, 2020, 13.

33. Shahzad, N.; Zegeye, H. Convergence theorems of common solutions for fixed point, variational inequality and equilibrium problems, J. Nonlinear Var. Anal. 2019, 3, 189-203.

34. Farid, M. The subgradient extragradient method for solving mixed equilibrium problems and fixed point problems in Hilbert spaces. J. Appl. Numer. Optim. 2019, 1, 335-345.

35. Flåm, S.D.; Antipin, A.S. Equilibrium programming using proximal-like algorithms. Math. Program. 1996, 78, 29-41. [CrossRef]

36. Korpelevich, G. The extragradient method for finding saddle points and other problems. Matecon 1976, 12, 747-756.

37. Yang, J.; Liu, H.; Liu, Z. Modified subgradient extragradient algorithms for solving monotone variational inequalities. Optimization 2018, 67, 2247-2258. [CrossRef]

38. Vinh, N.T.; Muu, L.D. Inertial Extragradient Algorithms for Solving Equilibrium Problems. Acta Math. Vietnam. 2019, 44, 639-663. [CrossRef] 
39. Hieu, D.V.; Cho, Y.J.; bin Xiao, Y. Modified extragradient algorithms for solving equilibrium problems. Optimization 2018, 67, 2003-2029. [CrossRef]

40. Bianchi, M.; Schaible, S. Generalized monotone bifunctions and equilibrium problems. J. Optim. Theory Appl. 1996, 90, 31-43. [CrossRef]

41. Mastroeni, G. On Auxiliary Principle for Equilibrium Problems. In Nonconvex Optimization and Its Applications; Springer: New York, NY, USA, 2003; pp. 289-298. [CrossRef]

42. Kreyszig, E. Introductory Functional Analysis with Applications, 1st ed.; Wiley Classics Library, Wiley: Hoboken, NJ, USA, 1989.

43. Tiel, J.V. Convex Analysis: An Introductory Text, 1st ed.; Wiley: New York, NY, USA, 1984.

44. Ioffe, A.D.; Tihomirov, V.M. (Eds.) Theory of Extremal Problems. In Studies in Mathematics and Its Applications 6; North-Holland, Elsevier: Amsterdam, thte Netherlands; New York, NY, USA, 1979.

45. Opial, Z. Weak convergence of the sequence of successive approximations for nonexpansive mappings. Bull. Amer. Math. Soc. 1967, 73, 591-598. [CrossRef]

46. Tan, K.; Xu, H. Approximating Fixed Points of Nonexpansive Mappings by the Ishikawa Iteration Process. J. Math. Anal. Appl. 1993, 178, 301-308. [CrossRef]

47. Bauschke, H.H.; Combettes, P.L. Convex Analysis and Monotone Operator Theory in Hilbert Spaces; Springer: New York, NY, USA, 2011; Volume 408.

48. Browder, F.; Petryshyn, W. Construction of fixed points of nonlinear mappings in Hilbert space. J. Math. Anal. Appl. 1967, 20, 197-228. [CrossRef]

(C) 2020 by the authors. Licensee MDPI, Basel, Switzerland. This article is an open access article distributed under the terms and conditions of the Creative Commons Attribution (CC BY) license (http://creativecommons.org/licenses/by/4.0/). 